\title{
A comparative study of the Bivalvia (Mollusca) from the continental shelves of Antarctica and Brazil
}

\author{
Flávio Dias Passos $^{1,2}$ \& Frederico Thomaisino Magalhães ${ }^{1}$ \\ ${ }^{1}$ Department of Animal Biology, Institute of Biology, University of Campinas - UNICAMP, \\ P.O. Box 6109, CEP 13083-970, Campinas, SP, Brazil \\ ${ }^{2}$ Corresponding author: Flávio Dias Passos, e-mail: flaviodp@unicamp.br
}

PASSOS, F.D. \& MAGALHÃES, F.T. A comparative study of the Bivalvia (Mollusca) from the continental shelves of Antarctica and Brazil. Biota Neotrop. 11(1): http://www.biotaneotropica.org.br/v11n1/en/ abstract?article+bn02211012011.

\begin{abstract}
During identification of bivalve molluscs collected in Antarctica, a rich taxonomic bibliography was gathered, stimulating comparisons with the Brazilian malacofauna. We listed a total of 68 and 368 known shallow-water species (less than $200 \mathrm{~m}$ depth) from Antarctica and Brazil, respectively, in order to find species, families and superfamilies in common, and to investigate how these malacofaunas differ in regard to these representative groups and their life habits. There are 23 superfamilies absent in Antarctica, but present in Brazil with at least one species; the reverse does not occur, as all superfamilies known from Antarctica are also recorded from Brazil. The number of Brazilian species is higher, being composed of a mixture of taxa from different biogeographical provinces, whereas in Antarctica there are only a few species adapted to its polar conditions, with minor components from elsewhere. Thus, many typical Caribbean species extend into Brazil, belonging to the diverse Arcoidea, Pectinoidea, Lucinoidea, Cardioidea, Veneroidea, and Tellinoidea. Cemented Ostreoidea, Plicatuloidea, Dimyoidea, Spondylidae (Pectinoidea), and Chamoidea are absent from Antarctica, as are wood (Teredinidae, Pholadoidea) and rock borers (Pholadidae, Pholadoidea; Gastrochaenoidea; and Lithophaginae, Mytiloidea). A large number of Brazilian species of infaunal (e.g., Tellinidae, Veneridae, Cardiidae, and Mactroidea) and epifaunal groups (Pectinidae, Mytilidae, and Arcidae) are absent from or poorly represented in Antarctica. Nuculanoidea, Limopsoidea, Lucinoidea, Galeommatoidea, Cyamioidea, and Cuspidarioidea are the richest groups in Antarctica; some of them are also represented by several species in Brazil, albeit in deeper waters. Three species are recorded as living in both places: Limatula pygmaea (Limidae), Lasaea adansoni (Lasaeidae), and Gaimardia trapesina (Gaimardiidae). Through the analysis of these groups from each fauna, it is possible to identify those that are taxonomically diverse in one place or another, and then emphasize them in ecological studies, eventually using them as model or monitoring organisms. The present paper can be a starting point for future discussion on the existing latitudinal gradients along the coast of eastern South America, stimulating studies on changes occurring in the composition of the faunas of bivalves from Brazil, Uruguay, Argentina, and Antarctica.
\end{abstract}

Keywords: checklist, shallow waters, brazilian fauna, antarctic fauna.

PASSOS, F.D. \& MAGALHÃES, F.T. Estudo comparativo dos Bivalvia (Mollusca) da plataforma continental da Antártica e do Brasil. Biota Neotrop. 11(1): http://www.biotaneotropica.org.br/v11n1/pt/ abstract?article+bn02211012011.

Resumo: Durante a identificação de moluscos bivalves coletados na Antártica, foi reunida uma rica bibliografia taxonômica, estimulando comparações com a malacofauna do Brasil. Assim, listamos um total de 68 espécies conhecidas para águas rasas (menos de 200 m de profundidade) da Antártica e 368 para o Brasil, procurando encontrar espécies, famílias e superfamílias em comum a ambos os locais, e investigando em que essas malacofaunas diferem em relação aos grupos representados e em relação ao hábito de vida das suas espécies. Vinte e três superfamílias não possuem representantes antárticos, mas estão presentes com pelo menos uma espécie brasileira; o oposto não ocorre, pois todas superfamílias que ocorrem na Antártica também são conhecidas para o Brasil. O número de espécies brasileiras é maior, composto por uma mistura de táxons de diferentes províncias biogeográficas, enquanto na Antártica existem somente poucas espécies adaptadas às condições polares, com uma minoria de representantes de fora da Antártica. Dessa forma, muitas espécies típicas do caribe se distribuem até o Brasil, pertencendo aos diversos Arcoidea, Pectinoidea, Lucinoidea, Cardioidea, Veneroidea e Tellinoidea. Cimentantes Ostreoidea, Plicatuloidea, Dimyoidea, Spondylidae (Pectinoidea) e Chamoidea não estão presentes na Antártica, como também não estão perfuradores de madeira (Teredinidae, Pholadoidea) e de rochas (Pholadidae, Pholadoidea; Gastrochaenoidea; e Lithophaginae, Mytiloidea). É notável o grande número de espécies brasileiras de grupos infaunais (exemplos, Tellinidae, Veneridae, Cardiidae e Mactroidea) e epifaunais (Pectinidae, Mytilidae e Arcidae), que são ausentes ou pobremente representados na Antártica. Nuculanoidea, Limopsoidea, Lucinoidea, Galeommatoidea, Cyamioidea e Cuspidarioidea são os grupos mais ricos em espécies antárticas, alguns deles também sendo especiosos no Brasil, entretanto, em maiores profundidades. Três espécies são registradas para ambos os locais: Limatula pygmaea (Limidae), Lasaea adansoni (Lasaeidae) e Gaimardia trapesina (Gaimardiidae). Através de análises dos grupos, é possível apontar aqueles que são taxonomicamente diversos em uma ou outra fauna, e então enfatizá-los em estudos ecológicos, utilizando-os como "organismos monitores" ou modelos. O presente trabalho pode ser um ponto de partida para futuras discussões sobre a ocorrência de um gradiente latitudinal ao longo da costa leste da América do Sul, estimulando trabalhos sobre mudanças que ocorrem na composição das faunas de bivalves do Brasil, Uruguai, Argentina e Antártica.

Palavras-chave: lista de espécies, águas rasas, fauna brasileira, fauna antártica. 


\section{Introduction}

The importance of studies of Antarctic organisms has been recently acknowledged. With recognition of global-scale phenomena such as, for example, those related to climate changes, knowledge of these animals has become essential for better comprehension and proper management of ecosystems, raising questions about their ecology and physiology. These studies are largely dependent on the correct identification of the species, eventually stimulating investigations on taxonomy, systematics, and biogeography.

The Brazilian Antarctic Program (PROANTAR) is contributing to knowledge of the organisms from the South Shetland Islands (an archipelago near the Antarctic Peninsula), especially of those from the coastal areas around King George Island, where the program's scientific activities are now concentrated. Beginning with the first Brazilian expeditions, many molluscs have been collected from this location, and brought for identification by the malacologists of the Instituto de Biociências da Universidade de São Paulo (IBUSP), coordinated by the late Prof. Walter Narchi (Narchi et al. 2002, Domaneschi et al. 2007). Investigations on the biology and anatomy of bivalves were continued by Prof. Osmar Domaneschi and his students (Narchi et al. 2002, Domaneschi et al. 2002, 2007, Passos et al. 2005, 2007, Sartori \& Domaneschi 2005, Passos \& Domaneschi 2006, 2009, Sartori et al. 2006), who gathered a rich bibliography on these animals, especially on their taxonomy.

Through a series of papers on the Antarctic bivalves, Nicol pointed out some of their peculiar characteristics, such as the high percentage of small-sized species (less than $15.0 \mathrm{~mm}$ ) (Nicol 1964b, 1966a); the small number of species of some groups that are otherwise well represented in warm shallow waters (venerids, lucinids, tellinids, and cardiids) (Nicol \& Gavenda 1964); the lack of shell-attached species (Nicol 1964a); some morphological characters which are common, such as thin and chalky shells, with a lack of bright colors and absence of color patterns and spines, and ornamentation, when present, subdued (Nicol 1965, 1967, 1970); and the low percentage of Antarctic species with infaunal habits (Nicol 1970). Subsequently, the evolutionary history of the Antarctic Bivalvia was analyzed (Crame 1992, 1996, 1997, 2000), as well as other aspects, including biogeography (Crame 1993, Brandt et al. 1999), physiology (e.g., Clarke 1983, Ahn 1997, Peck \& Conway 2000, Abele et al. 2001, Heilmayer \& Brey 2003), ecology (e.g., Ralph \& Maxwell 1977, Stockton 1984, Berkman 1990, Arnaud \& Hain 1992, Brey \& Hain 1992, Brey et al. 1993, Nigro 1993, Peck \& Bullough 1993, Cattaneo-Vietti et al. 1997, 2000, Mercuri et al. 1998, Urban \& Mercuri 1998, Chiantore et al. 2003), and reproductive biology (e.g., Richardson 1980, Berkman et al. 1991, Hain \& Arnaud 1992, Prezant et al. 1992, Bigatti et al. 2001, Peck et al. 2007, Kang et al. 2009, Passos \& Domaneschi 2009). In the present contribution, the faunas of Bivalvia from Brazil and Antarctica were compared, aiming to answer the following questions: At higher taxonomic levels (families, superfamilies), which taxa mostly diverge? Are there species that occur in both places? What are their differences in life habits? And finally, what are the possible causes of these divergences? These questions were investigated here, through examination of the species on the continental shelf, excluding those from deeper waters.

\section{Materials and Methods}

Initially, a comparison between the Antarctic and Brazilian bivalves at the species and genus levels appeared to be a very difficult task, in view of the many existing taxonomic debates. Apart from problems related to synonymy, the relationships of some genera are still unresolved, awaiting worldwide revisions. For example, some authors consider that Tellina and Thyasira are composed by many subgenera (e.g., Mikkelsen \& Bieler 2008), while others split them into many genera (e.g., Rios 1994, 2009, Coan et al. 2000). These subjects are not treated here. Although fewer, there are also taxonomic debates at the family and superfamily levels, and to facilitate comparisons, some decisions were taken, as follows.

The family Manzanellidae was considered in a separate superfamily, the Manzanelloidea, apart from the Solemyoidea. Glycymerididae was included within the Arcoidea. Plicatulidae was separated from Dimyidae, each forming its own superfamily, Plicatuloidea and Dimyoidea. One single group, Crassatelloidea, comprises four families (Crassatellidae, Astartidae, Carditidae, and Condylocardiidae), and five others (Galeommatidae, Lasaeidae, Leptonidae, Kelliidae, and Chlamydoconchidae) compose the Galeommatoidea. Trapezidae is placed in Arcticoidea, and Glossidae, Kelliellidae, and Vesicomyidae are grouped in Glossoidea. Cyamiidae, Neoleptonidae, Gaimardiidae, and Sportellidae form the Cyamioidea. Gastrochaenidae was treated in a separate superfamily, the Gastrochaenoidea. The Thracioidea were removed from the Pandoroidea, with the former composed by Thraciidae, Laternulidae, and Periplomatidae, and the latter by Pandoridae and Lyonsiidae. And finally, the septibranch families are grouped into three distinct superfamilies: Poromyoidea (Poromyidae), Cuspidarioidea (Cuspidariidae), and Verticordioidea (Verticordiidae and Lyonsiellidae).

Therefore, with these taxonomic problems in mind, an extensive bibliographic survey was carried out, to list the species recorded from the shelf of the Antarctic and Brazilian coasts, and excluding groups exclusively found in fresh and deep water, or only represented by fossil species. Each species was carefully analyzed as to its bathymetric distribution, and only those recorded from less than 200 meters depth were listed, hence restricting our analysis to the nearshore fauna. For the Antarctic, we included only the species recorded from around the continent and the islands of the Scotia Arc (except South Georgia); species recorded only from other subantarctic islands were excluded. For the Bivalvia living in Antarctica, the main references were Pelseneer (1903), Lamy (1906a, b, 1911), Melvill \& Standen (1907), Thiele (1912), Smith (1915), Hedley (1916), Carcelles (1944, 1950, 1953), Carcelles \& Williamson (1951), Powell (1951, 1958, 1960), Soot-Ryen (1951), Stuardo (1962), Castellanos (1963), Dell (1964, 1990), Nicol (1966b), Arnaud (1972, 1973), Rabarts \& Whybrow (1979), Arnaud et al. (1986, 2001), Mühlenhardt-Siegel (1989), Hain (1990), Villarroel \& Stuardo (1998), Linse (1999, 2004, 2006), Forcelli (2000), Narchi et al. (2002), Zelaya \& Ituarte (2002, 2006), Griffiths et al. (2003), Passos et al. (2005, 2007), Sartori \& Domaneschi (2005), Zelaya (2005, 2009, 2010), Passos \& Domaneschi (2006), Aldea et al. (2008, 2009), Aldea \& Troncoso (2008), and Troncoso \& Aldea (2008). For Brazil, the references were Klappenbach (1965, 1966a, b), Boss (1966, 1968, 1969, 1972), Narchi (1966, 1973, 1974, 1975, 1976, 1985a, b), Matthews \& Kempf (1970), Morrison (1971), Penna (1971), Abbott (1974), Marini (1974), Coelho \& Campos (1975), Narchi \& Domaneschi (1977), Penna-Neme (1978, 1983), Domaneschi (1984, 1985), Avelar \& Narchi (1984), Esteves (1984), Penna-Neme \& Cruz-Natali (1984), Tenório (1984), Domaneschi \& Lopes (1986a, b, 1988/9), Tenório et al. (1986), Guerón \& Coelho (1989), Lopes \& Narchi (1993), Rios (1994, 2009), Domaneschi \& Narchi (1998), Salvador et al. (1998), Simone (1998, 2001, 2008, 2009a, b), Domaneschi \& Martins (2002), Leonel et al. (2002), Quast (2003), Soares-Gomes \& Pires-Vanin (2003), Allen (2004), Domaneschi \& Shea (2004), Meserani et al. (2004), Simone \& Chichvarkhin (2004), Absalão \& Pimenta (2005), Arruda (2005), Arruda \& Domaneschi (2005), Souza et al. (2005), Amaral et al. (2006), Denadai et al. (2006), Simone \& Gonçalves (2006), Arruda et al. (2007), Mikkelsen 
\& Bieler (2008), Oliveira \& Absalão (2008), Resgalla et al. (2008), Simone \& Cunha (2008a, b), and Simone \& Penchaszadeh (2008).

Based on the literature, representative species of each family or superfamily were investigated regarding their life habits, in order to classify them as infaunal (I) or epifaunal (E). Additionally, the infaunal species were also classified as rock/wood borers (Ib) or tube dwellers (It), and the epifaunal as cemented to the substratum (Ec). Some groups are composed of species with different modes of life, and so are very diverse in this respect. These cases were also noted, with some caution in generalizations. For example, among the Arcoida and Limopsoidea, members of some families are epifaunal, living attached by byssus threads (Arcidae, Noetiidae, and Philobryidae); while for other families they are infaunal (Glycymerididae, Limopsidae). In the Mytiloidea, many Mytilinae species are byssally attached, but the Lithophaginae are rock borers. Within the Pectinoidea, species of Spondylidae are sessile, living cemented to the substratum, a life habit very different from the Pectinidae and Propeamussiidae. And finally, Galeommatoidea, Cyamioidea, and Myoidea are highly diverse, with both infaunal and epifaunal species. For this analysis, more general works were consulted (Boss 1982, Mikkelsen \& Bieler 2008, Beesley et al. 1998), as well as other papers on some species both from Antarctica and Brazil (cited above).

\section{Results}

The Bivalvia known from shallow waters of Antarctica and Brazil are listed as follows, totaling 68 and 368 species, respectively:

\section{Species from Antarctica}

\section{SUPERFAMILY NUCULOIDEA}

Family Nuculidae: Nucula falklandica Preston, 1912.

\section{SUPERFAMILY NUCULANOIDEA:}

Family Nuculanidae: Nuculana inaequisculpta (Lamy, 1906), Propeleda longicaudata (Thiele, 1912), Yoldiella antarctica (Thiele, 1912), Yoldiella valettei (Lamy, 1906), Yoldiella sabrina (Hedley, 1916), and Yoldiella profundorum (Melvill \& Standen, 1912);

Family Yoldiidae: Yoldia eightsi (Couthouy, 1839 in Jay);

Family Siliculidae: Silicula rouchi Lamy, 1910.

\section{SUPERFAMILY LIMOPSOIDEA:}

Family Limopsidae: Limopsis marionensis Smith, 1885, Limopsis lilliei Smith, 1915, Limopsis scotiana Dell, 1964, Limopsis scabra Thiele, 1912, and Limopsis enderbyensis Powell, 1958;

Family Philobryidae: Philobrya sublaevis Pelseneer, 1903, Philobrya wandelensis Lamy, 1906, Philobrya olstadi (SootRyen, 1951), Philobrya capillata Dell, 1964, Adacnarca nitens Pelseneer, 1903, Adacnarca limopsoides (Thiele, 1912), Lissarca miliaris (Philippi, 1845), and Lissarca notocardensis Melvill \& Standen, 1907.

Superfamily Mytiloidea:

Family Mytilidae: Dacrydium albidum Pelseneer, 1903.

\section{SUPERFAMILY LIMOIDEA:}

Family Limidae: Limatula pygmaea (Philippi, 1845), Limatula simillima Thiele, 1912, Limatula hodgsoni (Smith, 1907), and Limatula ovalis (Thiele, 1912).

\section{SUPERFAMILY PECTINOIDEA:}

Family Pectinidae: Adamussium colbecki (Smith, 1902);

Family Propeamussiidae: Cyclochlamys gaussianus (Thiele, 1912) and Cyclopecten pteriola (Melvill \& Staden, 1907).

\section{SUPERFAMILY LUCINOIDEA:}

Family Lucinidae: Epicodakia falklandica Dell, 1964;

Family Thyasiridae: Thyasira falklandica Smith, 1885, Thyasira bongraini (Lamy, 1910), Parathyasira dearborni (Nicol, 1965), Axinulus antarcticus Zelaya, 2010, and Genaxinus debilis Thiele, 1912 [Observation: Nicol (1966) and Hain (1990) referred $G$. debilis to G. bongraini, and Zelaya (2005) noted that its presence is "uncertain" for South Georgia, South Orkneys, and the South Shetlands islands; Aldea et al. (2008) considered this species a synonym of $T$. dearborni].

\section{SUPERFAMILY CRASSATELLOIDEA:}

Family Astartidae: Astarte longirostris Orbigny, 1846;

Family Carditidae: Cyclocardia astartoides (Martens, 1878).

\section{SUPERFAMILY GALEOMMATOIDEA:}

Family Lasaeidae: Lasaea consaguinea (Smith, 1877), Lasaea adansoni (Gmelin, 1791), Mysella minuscula (Pfeffer, 1886), Mysella charcoti (Lamy, 1906), Mysella gibbosa (Thiele, 1912), Mysella narchii Passos \& Domaneschi, 2006, Mysella antarctica (Smith, 1907), Montacuta nimrodiana (Hedley, 1911), and Waldo parasiticus (Dall, 1876);

Family Kelliidae: Kellia simulans Hedley, 1911, Pseudokellya cardiformis Smith, 1885, and Pseudokellya gradata Thiele, 1912;

Family Leptonidae: Solecardia antarctica Hedley, 1911.

\section{SUPERFAMILY CYAMIOIDEA:}

Family Gaimardiidae: Kidderia subquadratum (Pelseneer, 1903) and Gaimardia trapesina (Lamarck, 1819);

Family Cyamiidae: Cyamiomactra laminifera (Lamy, 1906), Cyamiocardium denticulatum (Smith, 1907), Cyamiocardium crassilabrum Dell, 1964, and Ptychocardia vanhoeffeni Thiele, 1912;

Family Neoleptonidae: Neolepton parasiticum (Dall, 1876).

\section{SUPERFAMILY HIATELLOIDEA:}

Family Hiatellidae: Hiatella meridionalis Orbigny, 1846.

\section{SUPERFAMILY PANDOROIDEA:}

Family Lyonsiidae: Lyonsia arcaeformis Martens, 1885.

\section{SUPERFAMILY THRACIOIDEA:}

Family Thraciidae: Thracia meridionalis Smith, 1885;

Family Laternulidae: Laternula elliptica (King \& Broderip, 1831).

\section{SUPERFAMILY POROMYOIDEA:}

Family Poromyidae: Poromya adelaidis (Hedley, 1916).

\section{SUPERFAMILY CUSPIDARIOIDEA:}

Family Cuspidariidae: Cuspidaria infelix Thiele, 1912, Cuspidaria kerguelensis Smith, 1885, Cuspidaria tenella Smith, 1907, Cuspidaria minima (Egorova, 1993), and Cuspidaria concentrica Thiele, 1912.

\section{Species from Brazil}

\section{SUPERFAMILY SOLEMYOIDEA}

Family Solemyidae: Solemya patagonica Smith, 1885 and Solemya notialis Simone, 2009 [Observation: Rios (1994, 2009) and Mikkelsen \& Bieler (2008) referred S. notialis to S. occidentalis Deshayes, 1857, which was considered to be restricted to the Florida-Caribbean Region by Abbott (1974)].

\section{SUPERFAMILY MANZANELLOIDEA:}

Family Manzanellidae: Nucinella serrei Lamy, 1912. 


\section{SUPERFAMILY NUCULOIDEA:}

Family Nuculidae: Nucula brasiliana Esteves, 1984, Nucula semiornata Orbigny, 1846, Nucula venezuelana Weisbord, 1964 and Ennucula puelcha (Orbigny, 1846) [Observation: Abbott (1974) referred N. semiornata to N. crenulata Adams, 1856, a species recorded by Mikkelsen \& Bieler (2008) from "North Carolina to Florida, West Indies, Gulf of Mexico, Caribbean Central America, South America (to Patagonia)"].

\section{SUPERFAMILY NUCULANOIDEA:}

Family Nuculanidae: Nuculana acuta (Conrad, 1832), Nuculana cestrota (Dall, 1890), Nuculana concentrica (Say, 1824), Nuculana larranagai Klappenbach \& Scarabino, 1968, Nuculana fortiana Esteves, 1984, Nuculana platessa (Dall, 1889), Adrana electa (Adams, 1846), Adrana patagonica (Orbigny, 1846), and Adrana tellinoides (Sowerby, 1823);

Family Malletiidae: Malletia cumingii (Hanley, 1860);

Family Yoldiidae: Yoldia riograndensis Esteves, 1984, Orthoyoldia crosbyana (Guppy, 1882) and Orthoyoldia scapania (Dall, 1889);

Family Tindariidae: Tindaria striata (King, 1831).

\section{SUPERFAMILY ARCOIDEA:}

Family Arcidae: Arca imbricata Bruguière, 1789, Arca zebra (Swainson, 1833), Barbatia cancellaria (Lamarck, 1819), Barbatia candida (Helbling, 1779), Barbatia ectocomata (Dall, 1886), Barbatia tenera (Adams, 1845), Acar dominguensis (Lamarck, 1819), Acar transmar Simone, 2009, Anadara brasiliana (Lamarck, 1819), Anadara chemnitzi (Philippi, 1851), Anadara baughmani Hertlein, 1951, Anadara notabilis (Röding, 1798), and Lunarca ovalis (Bruguière, 1789);

Family Noetiidae: Noetia bisulcata (Lamarck, 1819) and Arcopsis adamsi (Dall, 1886);

Family Glycymerididae: Glycymeris longior (Sowerby, 1832), Glycymeris undata (Linnaeus, 1758), Glycymeris tellinaeformis (Reeve, 1843), Glycymeris decussata (Linnaeus, 1758) and Glycymeris pectinata (Gmelin, 1790).

\section{SUPERFAMILY LIMOPSOIDEA:}

Family Limopsidae: Limopsis aurita (Brocchi, 1814), Limopsis janeiroensis Smith, 1915, and Limopsis davinae Esteves, 1984;

Family Philobryidae: Cratis antillensis (Dall, 1881), Cosa brasiliensis Klappenbach, 1966, and Cosa caribaea Abbott, 1958.

\section{SUPERFAMILY MYTILOIDEA:}

Family Mytilidae: Mytilus edulis platensis Orbigny, 1846, Aulacomya ater (Molina, 1782), Brachidontes exustus (Linnaeus, 1758), Brachidontes solisianus (Orbigny, 1846), Brachidontes rodriguezi (Orbigny, 1846), Mytella charruana (Orbigny, 1846), Mytella guyanensis (Lamarck, 1819), Perna perna (Linnaeus, 1758), Modiolus americanus (Leach, 1815), Modiolus carvalhoi Klappenbach, 1966, Amygdalum dentriticum Muhlfeld, 1811, Amygdalum sagittatum Rehder, 1934, Lioberus castaneus (Say, 1822), Crenella decussata (Montagu, 1808), Botula fusca (Gmelin, 1791), Gregariella coralliophaga (Gmelin, 1791), Musculus lateralis (Say, 1822), Musculus viator (Orbigny, 1846), Lithophaga antillarum (Orbigny, 1853), Lithophaga nigra (Orbigny, 1842), Lithophaga bisulcata (Orbigny, 1853), Lithophaga patagonica (Orbigny, 1847), and Myophorceps aristatus (Dillwyn, 1847) [Observations: Avelar \& Narchi (1984) referred B. exustus to B. darwinianus darwinianus (Orbigny, 1846). Klappenbach (1965) recorded M. charruana as M. falcata (Orbigny, 1846). Klappenbach (1965) and Rios (1994) referred $C$. decussata to $C$. divaricata (Orbigny, 1846), which was considered a synonym of it by Mikkelsen \& Bieler (2008)].

\section{SUPERFAMILY PTERIOIDEA:}

Family Pteriidae: Pteria colymbus (Röding, 1798) and Pinctada imbricata Röding, 1798 [Observation: Rios (1994) considered $P$. colymbus as a synonym of $P$. hirundo (Linnaeus, 1758); we follow Domaneschi \& Lopes (1986a), considering Pteria colymbus as valid];

Family Malleidae: Malleus candeanus (Orbigny, 1842);

Family Isognomonidae: Isognomon bicolor (Adams, 1845) [Observation: Rios (1994) recorded this species as I. alatus (Gmelin, 1791), and Mikkelsen \& Bieler (2008) listed I. bicolor, I. alatus and I. radiatus (Anton, 1838) occurring in Brazil].

\section{SUPERFAMILY PINNOIDEA:}

Family Pinnidae: Pinna carnea Gmelin, 1791 and Atrina seminuda (Lamarck, 1819).

\section{SUPERFAMILY LIMOIDEA:}

Family Limidae: Lima caribaea Orbigny, 1853, Lima scabra (Born, 1778), Limaria albicoma (Dall, 1886), Limaria inflata Lamarck, 1819, Limaria locklini (Ginty, 1955), Limatula confusa (Smith, 1885), Limatula hendersoni Olsson \& McGinty, 1958, Limatula pygmaea (Philippi, 1845), Limatula laminifera (Smith, 1885) and Limea bronniana Dall, 1886 [Observations: Abbott (1974) referred L. caribaea as a subspecies of L. lima (Linnaeus, 1758 ) and Rios (1994) considered that L. caribaea is a synonym of L. lima; we follow Mikkelsen \& Bieler (2008), who considered L. lima a species restricted to the Mediterranean Sea. Abbott (1974) recorded that L. inflata is often referred to as L. pellucida Adams, 1846, which was recorded by Mikkelsen \& Bieler (2008) as occurring from "North Carolina to Florida, Bermuda, Bahamas, West Indies, Gulf of Mexico, Caribbean Central America, South America (Brazil)"].

\section{SUPERFAMILY OSTREOIDEA:}

Family Gryphaeidae: Hyotissa macgintyi (Harry, 1985) [Observation: According to L.R.L. Simone (pers. com.), Rios (2009) referred this species to Pycnodonte hiotis (Linnaeus, 1758), which was introduced into the Caribbean Sea, but does not reach the Brazilian coast];

Family Ostreidae: Ostrea cristata Born, 1778, Ostrea equestris Say, 1834, Ostrea puelchana Orbigny, 1842, Crassostrea rhizophorae (Guilding, 1828), Crassotrea virginica (Gmelin, 1791), Crassostrea brasiliana (Lamarck, 1819), and Lopha frons (Linnaeus, 1758) [Observation: Rios (1994, 2009) considered that $C$. virginica does not occur in Brazil, but Mikkelsen \& Bieler (2008) recorded it from the entire western Atlantic. Rios (1994, 2009) referred $O$. brasiliana as a synonym of $C$. rhizophorae].

\section{SUPERFAMILY PLICATULOIDEA:}

Family Plicatulidae: Plicatula gibbosa Lamarck, 1801.

\section{SUPERFAMILY DIMYOIDEA:}

Family Dimyidae: Dimya argentea Dall, 1886.

\section{SUPERFAMILY PECTINOIDEA:}

Family Pectinidae: Amusium papyraceum (Gabb, 1873), Chlamys munda (Reeve, 1853), Chlamys felipponei (Dall, 1922), Chlamys multisquamata (Dunker, 1864), Chlamys muscosus (Wood, 1828), Chlamys ornata (Lamarck, 1819), Chlamys patagonica (King \& Broderip, 1832), Chlamys sentis (Reeve, 1853), Aequipecten tehuelchus (Orbigny, 1846), Leptopecten bavayi (Dauntzenberg, 1900), 
Argopecten gibbus (Linnaeus, 1758), Argopecten noronhensis (Smith, 1885), Nodipecten nodosus (Linnaeus, 1758), Pecten chazaliei Dautzenberg, 1900, Euvola ziczac (Linnaeus, 1758), and Cyclopecten subimbrifer Verrill \& Bush, 1897 [Observations: Mikkelsen \& Bieler (2008) referred C. munda to Spathochlamys benedicti (Verrill \& Bush, 1897). Rios (2009) considered $S$. benedicti a synonym of $C$. munda];

Family Propeamussiidae: Similipecten nanus (Verrill \& Bush, 1897) and Parvamussium pourtalesianum (Dall, 1886) [Observation: Rios (2009) did not record S. nanus from Brazil];

Family Spondylidae: Spondylus americanus Hermann, 1781, Spondylus erinaceus Reeve, 1856, and Spondylus ictericus Reeve, 1856.

\section{SUPERFAMILY ANOMIOIDEA:}

Family Anomiidae: Anomia simplex Orbigny, 1853 and Pododesmus rudis (Broderip, 1834).

\section{SUPERFAMILY LUCINOIDEA:}

Family Lucinidae: Lucina pectinata (Gmelin, 1791), Lucina muricata (Spengler, 1798), Lucina blanda (Dall \& Simpson, 1901), Lucina sombrerensis Dall, 1886, Callucina keenae Chavan, 1971, Codakia costata (Orbigny, 1842), Codakia orbicularis (Linnaeus, 1758), Codakia orbiculata (Montagu, 1808), Codakia pectinella (Adams, 1852), Linga amiantus (Dall, 1901), Myrtea lens (Verrill \& Smith, 1880), Miltha childrenae (Gray, 1824), and Divaricella quadrisulcata (Orbigny, 1845) [Observations: Abbott (1974) referred C. keenae to Parvilucina multilineata (Tuomey \& Holmes, 1857) and Rios (1994, 2009) to Lucina multilineata, but Mikkelsen \& Bieler (2008) pointed out that "multilineata Tuomey Holmes, 1857" is an invalid name. Penna-Neme \& Cruz-Natali (1984) referred $M$. lens to Pseudomiltha tixieri Klein, 1967, which was considered as a synonym of it by Rios $(1994,2009)]$;

Family Thyasiridae: Thyasira croulinensis Jeffreys, 1874 and Thyasira trisinuata (Orbigny, 1853);

Family Ungulinidae: Diplodonta danieli Klein, 1967, Diplodonta nucleiformis Wagner, 1838, Diplodonta patagonica (Orbigny, 1842), Diplodonta punctata (Say, 1822), Felaniella candeana (Orbigny, 1842), Felaniella vilardeboana (Orbigny, 1846), Phlyctiderma semiaspera (Philippi, 1836), and Timothynus rehderi (Altena, 1968).

\section{SUPERFAMILY CRASSATELLOIDEA:}

Family Crassatellidae: Crassatella riograndensis Vokes, 1973, Crassatella brasiliensis (Dall, 1903), Crassinella lunulata (Conrad, 1834), Crassinella marplatensis Castellanos, 1970, and Crassinella martinensis (Orbigny, 1842);

Family Carditidae: Carditamera floridana Conrad, 1838, Carditamera micella Penna, 1971, Carditamera plata (Ihering, 1907), Cyclocardia moniliata (Dall, 1902), and Pleuromeris sanmartini Klappenbach, 1971 [Observations: Rios (2009) did not record $C$. micella and C. plata from the Brazillian coast];

Family Condylocardiidae: Americuna besnardi Klappenbach, 1962 and Carditopsis smithi (Dall, 1896).

\section{SUPERFAMILY CHAMOIDEA:}

Family Chamidae: Chama congregata Conrad, 1833, Chama floridana Lamarck, 1819, Chama macerophylla Gmelin, 1791, Chama sarda Reeve, 1847, Chama sinuosa Broderip, 1835, Pseudochama radians (Lamarck, 1819), Arcinella arcinella (Linnaeus, 1767), and Arcinella brasiliana (Nicol, 1953).

\section{SUPERFAMILY GALEOMMATOIDEA:}

Family Galeommatidae: Parabornia palliopapillata Simone, 2001;
Family Lasaeidae: Lasaea adansoni (Gmelin, 1791); Family Kelliidae: Kellia suborbicularis (Montagu, 1803); Family Leptonidae: Lepton cema (Narchi, 1966);

Family Chlamydoconchidae: Chlamydoconcha avalvis Simone, 2008 [Observation: Rios (2009) did not record this species from the Brazillian coast].

SUPERFAMILY ARCTICOIDEA:

Family Trapezidae: Coralliophaga coralliophaga (Gmelin, 1791).

\section{SUPERFAMILY GLOSSOIDEA:}

Family Glossidae: Meiocardia agassizzii Dall, 1889;

Family Vesicomyidae: Vesicomya albida (Dall, 1889).

\section{SUPERFAMILY CYAMIOIDEA:}

Family Gaimardiidae: Gaimardia trapesina (Lamarck, 1819); Family Sportellidae: Basterotia quadrata (Hinds, 1843).

\section{SUPERFAMILY CARDIOIDEA:}

Family Cardiidae: Trachycardium magnum (Linnaeus, 1758), Trachycardium muricatum (Linnaeus, 1758), Trachycardium manueli Prado, 1993, Papyridea semisulcata (Gray, 1825), Papyridea soleniformis (Bruguière, 1789), Trigoniocardia antillarum (Orbigny, 1853), Americardia media (Linnaeus, 1758), Nemocardium peramabile (Dall, 1881), Nemocardium tinctum (Dall, 1881), Laevicardium brasilianum (Lamarck, 1819), and Laevicardium pictum (Ravenel, 1861) [Observations: Arruda (2005) considered T. manueli as a valid species, but Rios (2009) recorded it as a synonym of T. muricatum. Abbott (1974) referred L. brasilianum as L. laevigatum, which is considered a synonym of it by Rios (2009)];

\section{SUPERFAMILY VENEROIDEA:}

Family Veneridae: Ventricolaria foresti Fischer-Pietti \& Testud, 1967, Ventricolaria rigida (Dillwyn, 1817), Ventricolaria strigillina (Dall, 1902), Gouldia cerina (Adams, 1845), Chione cancellata (Linnaeus, 1767), Chione subrostrata (Lamarck, 1818), Chione intapurpurea (Conrad, 1849), Chione pubera (Bory Saint-Vicent, 1827), Chione latilirata (Conrad, 1841), Chione paphia (Linnaeus, 1767), Anomalocardia brasiliana (Gmelin, 1791), Clausinella gayi (Hupe, 1854), Protothaca antiqua (King \& Broderip, 1832), Protothaca pectorina (Lamarck, 1818), Tivela foresti FischerPiette \& Testud, 1967, Tivela fulminata (Valenciennes, 1827), Tivela mactroides (Born, 1778), Tivela ventricosa (Gray, 1838), Tivela isabelleana (Orbigy, 1846), Transenella cubaniana (Orbigny, 1853), Transennella stimpsoni Dall, 1902, Pitar fulminatus (Menke, 1828), Pitar palmeri (Fischer-Pietti \& Testud, 1967), Pitar rostratus (Koch, 1844), Pitar circinatus (Born, 1778), Pitar cordatus (Schwengel, 1951), Amiantis purpuratus (Lamarck, 1818), Callista maculata (Linnaeus, 1758), Callista eucymata (Dall, 1890), Transenpitar americana (Duello-Jurado, 1951), Eurhomalea exalbida (Dillwyn, 1817), Dosina concentrica (Born, 1778), and Cyclinella tenuis (Récluz, 1852) [Observation: Abbott (1974) referred Chione subrostrata as a synonym of $C$. cancellata, which was considered by Mikkelsen $\&$ Bieler (2008) as restricted to the Florida Keys (southeastern United States) and the eastern Caribbean];

Family Petricolidae: Petricola lapicida (Gmelin, 1791), Petricola stellae Narchi, 1975, Choristodon robustus (Sowerby, 1834), and Cooperella atlantica Rehder, 1943 [Observation: Abbott (1974) referred Choristodon robustus to Rupellaria typica (Jonas, 1844), and Narchi (1974) and Rios (1994, 2009) to 
Petricola typica; Mikkelsen \& Bieler (2008) considered R. typica as a synonym of $R$. robustus].

\section{SUPERFAMILY TELLINOIDEA:}

Family Tellinidae: Tellina radiata Linnaeus, 1758, Tellina petitiana Orbigny, 1846, Tellina iheringi Dall, 1900, Tellina diantha Boss, 1964, Tellina euvitrea Boss, 1964, Tellina exerythra Boss, 1964, Tellina gibber Ihering, 1907, Tellina probina Boss, 1964, Tellina sybaritica Dall, 1881, Tellina versicolor Kay, 1843, Tellina americana Dall, 1900, Tellina alternata Say, 1822, Tellina angulosa Gmelin, 1791, Tellina lineata Turton, 1819, Tellina nitens Adams, 1845, Tellina punicea Born, 1778, Tellina trinitatis (Tomlin, 1929), Tellina vespuciana Orbigny, 1842, Tellina aequistriata Say, 1824, Tellina alerta Boss, 1964, Tellina juttingae (Altena, 1965), Tellina martinicensis Orbigny, 1853, Tellina persica Dall \& Simpson, 1901, Tellina sandix Boss, 1968, Tellina listeri Röding, 1798, Strigilla carnaria (Linnaeus, 1758), Strigilla gabbi Olsson \& McGinty, 1958, Strigilla producta Tryon, 1870, Strigilla mirabilis (Philippi, 1841), Strigilla pisiformis (Linnaeus, 1758), Macoma cleryana (Orbigny, 1846), Macoma biota Arruda \& Domaneschi, 2006, Macoma pseudomera Dall \& Simpson, 1900, Macoma tenta (Say, 1834), Macoma constricta (Bruguière, 1792), Macoma brevifrons (Say, 1834), Macoma tageliformis Dall, 1900, Macoma uruguayensis (Smith, 1885), Macoma mitchelli Dall, 1895, Cymatoica orientalis (Dall, 1890), and Temnoconcha brasiliana (Dall, 1921) [Observation: Boss (1966), Tenório (1984), Arruda (2005), and Amaral et al. (2006) considered T. iheringi as a valid species, but Rios (1994, 2009) recorded it as a synonym of T. petitiana];

Family Donacidae: Donax gemmula Morrison, 1971, Donax hanleyanus Philippi, 1842, Donax striatus Linnaeus, 1767, Donax denticulatus Linnaeus, 1758, and Iphigenia brasiliana (Lamarck, 1818);

Family Psammobiidae: Asaphis deflorata (Linnaeus, 1758), Gari linhares Simone, 1998, Heterodonax bimaculatus (Linnaeus, 1758), Sanguinolaria sanguinolenta (Gmelin, 1791), and Sanguinolaria cruenta (Lightfoot, 1786);

Family Semelidae: Semele bellastriata (Conrad, 1937), Semele casali Doello-Jurado, 1949, Semele proficua (Pulteney, 1799), Semele purpurascens (Gmelin, 1791), Semele nuculoides (Conrad, 1841), Abra aequalis (Say, 1822), Abra lioica (Dall, 1881), Abra uruguayensis (Pilsbry, 1897), Cumingia coarctata Sowerby, 1833, Ervilia concentrica (Holmes, 1858), Ervilia nitens (Montagu, 1808), and Ervilia subcancellata Smith, 1885 [Observation: Rios (2009) considered E. concentrica and E. subcancellata as synonyms of E. nitens];

Family Solecurtidae: Solecurtus cumingianus (Dunker, 1861), Solecurtus sanctaemarthae Orbigny, 1846, Tagelus plebeius (Lightfoot, 1786), and Tagelus divisus (Spengler, 1794);

\section{SUPERFAMILY SOLENOIDEA:}

Family Solenidae: Solen tehuelchus Orbigny, 1843 and Solen obliquus Spengler, 1794;

\section{SUPERFAMILY MACTROIDEA:}

Family Mactridae: Mactra isabelleana Orbigny, 1846, Mactra marplatensis Doello-Jurado, 1918, Mactra patagonica Orbigny, 1846, Mactra fragilis Gmelin, 1791, Mactra iheringi Daft, 1897, Mactra petiti Orbigny, 1846, Mactra janeiroensis Smith, 1915, Mactrellona alata (Spengler, 1802), Mulinia cleryana (Orbigny, 1846), Anatina anatina (Spengler, 1802), and Raeta plicatella (Lamarck, 1818) [Observation: Rios (2009) referred Mactra marplatensis as a synonym of M. isabelleana];
Family Mesodesmatidae: Mesodesma mactroides Deshayes, 1854.

\section{SUPERFAMILY DREISSENOIDEA:}

Family Dreissenidae: Mytilopsis leucophaeta (Conrad, 1831).

\section{SUPERFAMILY MYOIDEA:}

Family Myidae: Sphenia antillensis Dall \& Simpson, 1901;

Family Corbulidae: Corbula caribaea Orbigny, 1842, Corbula lyoni Pilsbry, 1897, Corbula patagonica Orbigny, 1846, Corbula tryoni Smith, 1880, Corbula cymella Dall, 1881, Corbula dietziana Adams, 1852, Corbula cubaniana Orbigny, 1853, Corbula operculata Philippi, 1849, and Corbula tarasconii Arruda, Domaneschi, Francisco \& Barros, 2007 [Observation: Rios (2009) did not record C. tarasconii from the Brazilian coast].

\section{SUPERFAMILY HIATELLOIDEA:}

Family Hiatellidae: Hiatella solida (Sowerby, 1834) and Panopea abbreviata Valenciennes, 1839 [Narchi (1973) and Domaneschi \& Narchi (1998) recorded H. solida from Brazil, but Rios $(1994,2009)$ referred it to H. arctica (Linnaeus, 1767). Simone \& Penchaszadeh (2008) suggested that the Brazilian species is distinct from the European $H$. arctica and the Chilean H. solida, and Mikkelsen \& Bieler (2008) noted that "recent studies on Brazilian specimens of $H$. arctica show strong evidence of more than one species"].

\section{SUPERFAMILY GASTROCHAENOIDEA:}

Family Gastrochaenidae: Gastrochaena hians (Gmelin, 1791), Gastrochaena ovata Sowerby, 1834, and Spengleria rostrata (Spengler, 1783).

\section{SUPERFAMILY PHOLADOIDEA:}

Family Pholadidae: Pholas campechiensis Gmelin, 1791, Barnea lamellosa (Orbigny, 1946), Barnea truncata (Say, 1822), Cyrtopleura costata (Linnaeus, 1758), Cyrtopleura lanceolata (Orbigny, 1846), Martesia striata (Linnaeus, 1758), Martesia fragilis Verrill \& Bush, 1890, Martesia cuneiformis (Say, 1822), and Netastoma darwini (Sowerby, 1849);

Family Teredinidae: Teredo navalis Linnaeus, 1758, Teredo bartschi Clapp, 1923, Teredo fulleri Clapp, 1924, Teredo furcifera Martens, 1894, Teredo johnsoni Clapp, 1924, Teredo mindanensis Bartsch, 1923, Lyrodus floridanus (Bartsch, 1922), Lyrodus massa (Lamy, 1923), Lyrodus pedicellatus (Quatrefages, 1849), Neoteredo reynei (Bartsch, 1920), Bankia carinata (Gray, 1827), Bankia gouldi Bartsch, 1908, Bankia campanellata Moll \& Roch, 1931, Bankia destructa Clench \& Turner, 1946, Bankia bagidaesis Roch, 1929, Bankia cieba Clench \& Turner, 1946, Bankia fimbriatula Moll \& Roch, 1931, Bankia rochi Moll, 1931, Nausitora fusticula (Jeffreys, 1860), Nototeredo knoxi (Bartsch, 1917), and Psiloteredo healdi (Bartsch, 1931) [Observations: Abbott (1974) considered L. floridanus as a synonym of L. pedicellatus, and Rios $(1994,2009)$ did not record $P$. healdi from Brazil].

\section{SUPERFAMILY PHOLADOMYOIDEA:}

Family Pholadomyidae: Panacca arata (Verrill \& Smith, 1881).

\section{SUPERFAMILY PANDOROIDEA:}

Family Pandoridae: Pandora brasiliensis Sowerby, 1874 and Pandora bushiana Dall, 1886;

Family Lyonsiidae: Entodesma alvarezi Orbigny, 1846, Entodesma beana (Orbigny, 1853), and Entodesma patagonica (Orbigny, 1846) [Observation: Abbott (1974) recorded E. alvarezi as Lyonsia hyalina Conrad, 1831, which was considered as a synonym of it by Rios $(1994,2009)]$. 
SUPERFAMILY THRACIOIDEA:

Family Thraciidae: Thracia similis Couthouy, 1839 and Cyathodonta semirugosa (Reeve, 1859);

Family Periplomatidae: Periploma compressa Orbigny, 1846.

\section{SUPERFAMILY POROMYOIDEA:}

Family Poromyidae: Poromya cymata Dall, 1889, Poromya granulata (Nyst \& Westendorp, 1839), and Poromya elongata Dall, 1886.

\section{SUPERFAMILY CUSPIDARIOIDEA:}

Family Cuspidariidae: Cuspidaria braziliensis Smith, 1915, Cuspidaria platensis Smith, 1915, Cuspidaria rostrata (Spengler, 1793), Cardiomya cleryana (Orbigny, 1846), Cardiomya ornatissima (Orbigny, 1853), and Cardiomya striata (Jeffreys, 1876).

\section{SUPERFAMILY VERTICORDIOIDEA:}

Family Verticordiidae: Verticordia fischeriana Dall, 1881, Trigonulina ornata Orbigny, 1853, and Spinosipella tinga Simone \& Cunha, 2008 [Observation: Rios (2009) did not record T. ornata from Brazil].

In Table 1, all known extant superfamilies of marine Bivalvia are listed, including two (Trigonioidea and Clavagelloidea) that are recorded neither from Antarctica or Brazil. The mode of life and the total number of species counted for each taxa from both places are also shown.

There are 23 superfamilies that do not have Antarctic members, but are represented by at least one species in Brazil: Solemyoidea, Manzanelloidea, Arcoidea, Pterioidea, Pinnoidea, Ostreoidea, Plicatuloidea, Dimyoidea, Anomioidea, Chamoidea, Arcticoidea, Glossoidea, Cardioidea, Veneroidea, Tellinoidea, Solenoidea, Mactroidea, Dreissenoidea, Myoidea, Gastrochaenoidea, Pholadoidea, Pholadomyoidea, and Verticordioidea. The reverse does not occur, as all superfamilies occurring in Antarctica are also known from Brazil. Absent from Antarctica are the Arcoidea, Veneroidea, Tellinoidea and Pholadoidea (see also Figure 1), groups that comprise $42 \%$ of the total number of species recorded from Brazil. Mytiloidea is also characteristically rich in Brazilian coastal waters (23 species), but nearly absent from Antarctica (only one). Nuculanoidea, Limopsoidea, Galeommatoidea, and Cyamioidea are the superfamilies best represented in Antarctica (Table 1, Figure 1), comprising $60 \%$ of the total number of species. At the family level, the Antarctic harbors relatively more species of Philobryidae (8), Thyasiridae (5), Lasaeidae (9), Cyamiidae (4) and Cuspidariidae (5), groups that are represented by only a few species from shallow waters of Brazil. Only three species are recorded from both regions: Limatula pygmaea (Limidae), Lasaea adansoni (Lasaeidae), and Gaimardia trapesina (Gaimardiidae).

The mode of life for each superfamily is also shown in Table 1. In Antarctica, both infaunal (Nuculanoidea, Limopsidae, Thyasiridae, Cyamiidae, Cuspidariidae) and epifaunal (Philobryidae, Limidae) groups are diverse in number of species; but there are not members of the groups that are essentially sessile, living cemented to the substratum (Ostreoidea, Plicatuloidea, Dimyoidea, Spondylidae, Anomioidea, Chamoidea) or as wood or rock borers (Pholadoidea, Gastrochaenoidea, and species of Mytilidae and Petricolidae). In Brazil, the infaunal superfamilies are very well represented, including the Veneroidea, Tellinoidea and Lucinoidea; but wood borers (Pholadoidea) and epifaunal groups (Arcoidea, Mytiloidea, Pectinoidea) are also very diverse (Figure 1).
Table 1. Superfamilies of marine Bivalvia, with the number of species recorded from Antarctic and Brazilian shallow waters of the continental shelf $(<200 \mathrm{~m}$ depth). The life habits are classified as epifaunal (E) and infaunal (I), and further as cemented (Ec) and boring (Ib) or tube dwellers (It). See comments in the text for those superfamilies classified with two types of life habits.

Tabela 1. Superfamílias de bivalves marinhos, com o número de espécies registradas para águas rasas da plataforma continental $(<200 \mathrm{~m}$ de profundidade) da Antártica e do Brasil. Os modos de vida são classificados em epifaunal (E) e infaunal (I), e também como cimentados ao substrato (Ec), perfuradores (Ib) ou construidores de tubos (It). Ver comentários no texto para as superfamílias classificadas com dois modos de vida.

\begin{tabular}{|c|c|c|c|}
\hline \multirow[b]{2}{*}{ Superfamilies } & \multirow[b]{2}{*}{ Life habits } & \multicolumn{2}{|c|}{ Number of species } \\
\hline & & Antarctic & Brazil \\
\hline Solemyoidea & $\mathrm{I}$ & 0 & 2 \\
\hline Manzanelloidea & I & 0 & 1 \\
\hline Nuculoidea & $\mathrm{I}$ & 1 & 4 \\
\hline Nuculanoidea & $\mathrm{I}$ & 8 & 14 \\
\hline Arcoidea & $\mathrm{E} / \mathrm{I}$ & 0 & 20 \\
\hline Limopsoidea & $\mathrm{E} / \mathrm{I}$ & 13 & 6 \\
\hline Mytiloidea & $\mathrm{E} / \mathrm{Ib}$ & 1 & 23 \\
\hline Pterioidea & $\mathrm{E}$ & 0 & 4 \\
\hline Pinnoidea & I & 0 & 2 \\
\hline Limoidea & $\mathrm{E}$ & 4 & 10 \\
\hline Ostreoidea & $\mathrm{Ec}$ & 0 & 8 \\
\hline Plicatuloidea & $\mathrm{Ec}$ & 0 & 1 \\
\hline Dimyoidea & $\mathrm{Ec}$ & 0 & 1 \\
\hline Pectinoidea & $\mathrm{E} / \mathrm{Ec}$ & 3 & 21 \\
\hline Anomioidea & $\mathrm{E}$ & 0 & 2 \\
\hline Trigonioidea & I & 0 & 0 \\
\hline Lucinoidea & $\mathrm{I}$ & 6 & 23 \\
\hline Crassatelloidea & I & 2 & 12 \\
\hline Chamoidea & $\mathrm{Ec}$ & 0 & 8 \\
\hline Galeommatoidea & $\mathrm{E} / \mathrm{I}$ & 13 & 5 \\
\hline Arcticoidea & $\mathrm{I}$ & 0 & 1 \\
\hline Glossoidea & $\mathrm{I}$ & 0 & 2 \\
\hline Cyamioidea & $\mathrm{E} / \mathrm{I}$ & 7 & 2 \\
\hline Cardioidea & I & 0 & 11 \\
\hline Veneroidea & I & 0 & 37 \\
\hline Tellinoidea & I & 0 & 67 \\
\hline Solenoidea & I & 0 & 2 \\
\hline Mactroidea & $\mathrm{I}$ & 0 & 12 \\
\hline Dreissenoidea & $\mathrm{E}$ & 0 & 1 \\
\hline Myoidea & $\mathrm{E} / \mathrm{I}$ & 0 & 10 \\
\hline Hiatelloidea & $\mathrm{I}$ & 1 & 2 \\
\hline Gastrochaenoidea & $\mathrm{Ib}$ & 0 & 3 \\
\hline Pholadoidea & $\mathrm{Ib}$ & 0 & 30 \\
\hline Pholadomyoidea & I & 0 & 1 \\
\hline Pandoroidea & I & 1 & 5 \\
\hline Thracioidea & $\mathrm{I}$ & 2 & 3 \\
\hline Poromyoidea & $\mathrm{I}$ & 1 & 3 \\
\hline Cuspidarioidea & I & 5 & 6 \\
\hline Verticordioidea & $\mathrm{I}$ & 0 & 3 \\
\hline Clavagelloidea & It & 0 & 0 \\
\hline Total & & 68 & 368 \\
\hline
\end{tabular}




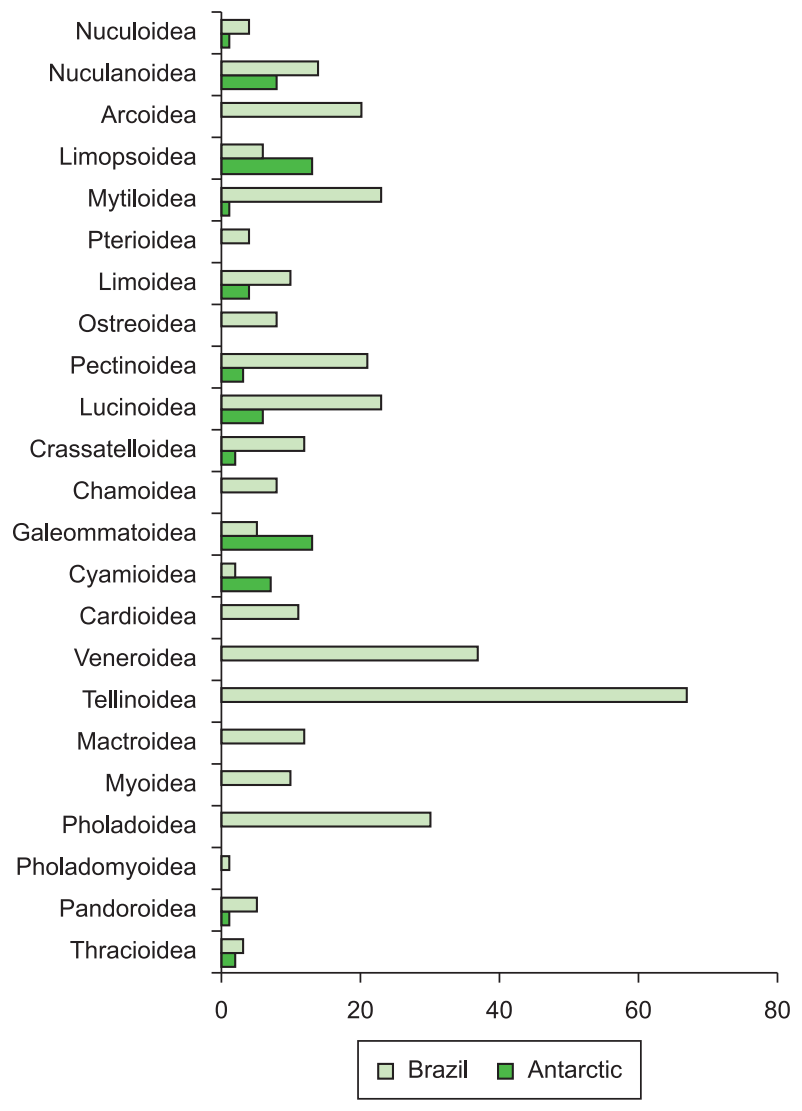

Figure 1. Main superfamilies of marine Bivalvia, with the number of species recorded from the Antarctic and Brazilian continental shelves ( $<200 \mathrm{~m}$ depth).

Figura 1. Principais superfamílias de bivalves marinhos, com o número de espécies registradas para águas da plataforma continental da Antártica e do Brasil ( $<200 \mathrm{~m}$ de profundidade).

\section{Discussion}

The total number of coastal Brazilian species is about five times the number recorded from the Antarctic, a proportion that appears to be unbalanced, as the latter has a more extensive coast related to the larger area of its continent. This can be explained in different ways, for example, by the greater homogeneity of the Antarctic marine environment, in general terms; whereas in Brazil there are different conditions along its coast. The Brazilian fauna is composed of a mixture of taxa from different provinces, with a strong contribution from the Caribbean region, which is considered a tropical high-diversity focus of marine bivalves (Crame 2000). From the Caribbean, many species of typical shallow-water groups have spread southward as far as the north/northeast Brazilian coast, for example the Arcoidea (e.g., Barbatia cancellaria and Glycymeris decussata), Pectinoidea (e.g., Pecten chazaliei, Spondylus americanus, and S. erinaceus), Lucinoidea (e.g., Lucina muricata), Cardioidea (e.g., Trigoniocardia antillarum and Nemocardium peramabile), Veneroidea (e.g., Chione intapurpurea, Transenella cubaniana, and Pitar cordatus), and Tellinoidea (e.g., Tellina euvitrea, T. probrina, T. sybaritica, T. americana, T. vespuciana, T. persica, Strigilla gabbi, S. mirabilis, Cymatoica orientalis, Donax striatus, D. denticulatus, and Cumingia coarctata). On the southernmost coast, the bivalve fauna has a different composition, with a subtropical subset more typical of the Argentinean-Magellanic province, including Solemya patagonica, Tindaria striata, Mytilus edulis platensis, Brachidontes rodriguezi, Chlamys felipponei, Clausinella gayi, Protothaca antiqua, Transenpitar americana, Eurhomalea exalbida, Mactra patagonica, Barnea lamellosa,
Netastoma darwini, Pandora brasiliensis, Entodesma patagonica, and Thracia similis. The Antarctic fauna, on the other hand, is composed mainly by circumantarctic species, adapted to a characteristic polar environment, with only a few members from other biogeographical provinces. Here, only some Magellanic species extend southward through the islands of the Scotia Arc into shallow waters of Antarctica, such as Nucula falklandica, Lissarca miliaris, Epicodakia falklandica, Thyasira falklandica, Waldo parasiticum, Kidderia subquadratum, and Hiatella meridionalis.

Comparing the Brazilian and Antarctic environments over a long time scale, the shallow waters of Antarctica have had their typical polar characteristics since the beginning of the Caenozoic, when the ice cap began to form. Since then, the scouring action of breaking ice has severely limited the survival of more sedentary coastal organisms, especially those from the intertidal zone, and probably also excluding sessile forms of life. This may be the cause of the absence of cemented groups of Bivalvia, such as Ostreoidea, Plicatuloidea, Dimyoidea, Spondylidae (Pectinoidea), and Chamoidea. Some species of Ostreoidea were present in Antarctic waters in past times, when conditions were milder (Crame 1996). Since mangroves and coral reefs are lacking in the cold shallow waters of Antarctica, there are no species of bivalves that are commonly associated with these ecosystems, such as wood borers (Teredinidae, Pholadoidea) and coral rock borers (Pholadidae, Pholadoidea; Gastrochaenoidea; and Lithophaginae, Mytiloidea). These clearly distinct conditions of the Antarctic and Brazilian waters also explain why their bivalve faunas are so different in terms of number of species, just related to the absence of important groups in Antarctica which are otherwise very rich in shallow Brazilian waters. Nicol (1967) already pointed out the absence of cemented, wood-boring, and rock-boring bivalves in Antarctica. In contrast, the Brazilian coast has 8 species of Ostreoidea and 30 of Pholadoidea.

Among the infaunal bivalve groups, there are large numbers of Brazilian species of Tellinoidea (67 spp.) and Veneroidea (37 spp.), contrasting with their complete absence in Antarctica. Within the American Quadrant, Macoma georgiana Dell, 1964 is the tellinoidean with the most southerly distribution, reaching South Georgia (Zelaya 2005), but not extending into the Scotia Arc and the Antarctic Peninsula. For the Veneroidea, three species are found in Patagonia and probably in the Falklands, but not in Antarctica: Eurhomalea exalbida (Dillwyn, 1817), Gomphina foveolata (Cooper \& Preston, 1910), and Protothaca antiqua (King \& Broderip, 1832). Powell (1960) noted the complete absence of Veneridae and Tellinidae, and also Cardiidae, from Antarctica. Apart from these infaunal groups, the Mactroidea is also lacking, whereas it is represented by 11 species in Brazil, similarly to the Cardioidea. The explanation for the absence of these important superfamilies in Antarctic waters is completely unknown, but may be related to some physiological or ecological factor, or to their evolutionary history. Crame (2000), for example, suggested that more recent families tend to exhibit stronger latitudinal gradients of diversity, decreasing in number of species from low latitudes toward the poles. According to this author, these gradients can be explained by the origin of some of these groups in tropical and low-latitude regions, which are now in the process of evolutionary radiation to the poles; Tellinidae, Veneridae, and Cardiidae were cited as examples.

Among the epifaunal superfamilies, Pectinoidea is represented by a small number of species in Antarctic shallow waters (only Adamussium colbecki and two species of Propeamussiidae), compared with 21 Brazilian species (16 Pectinidae, 2 Propeamussiidae, and 3 Spondylidae). More interesting, however, is the absence of Arcidae, in contrast to the 12 species recorded from Brazil. In fact, this family is represented by only one Antarctic species, Bathyarca sinuata (Pelseneer, 1903), which is found in deeper waters (400-2044 m) (Dell 1990, Aldea et al. 2008). Another similar case is Mytilidae, with two 
species in Antarctic waters, Dacrydium albidum and D. panamensis Knudsen, 1970, the former occurring in a wide depth range (122$4636 \mathrm{~m})$, and the latter restricted to deep waters (855-3770 m) (Knudsen 1970, Dell 1990, Aldea et al. 2008, Aldea \& Troncoso 2008). For both arcoids and mytiloids (but not for pectinids), Crame (2000) demonstrated that there is a tendency to increased diversity toward higher latitudes, suggesting an ongoing evolutionary radiation from the tropics to the poles, as it was hypothesized for Tellinidae, Veneridae, and Cardiidae.

Although represented by a small number of bivalve species (compared to Brazil), Antarctic shallow waters are rich in some groups, such as Nuculanoidea, Limopsoidea, Lucinoidea, Galeommatoidea, Cyamioidea, and Cuspidarioidea, with three of these even more speciose than in Brazil (Limopsoidea, Galeommatoidea, and Cyamioidea). Nicol (1970) already noted that more than one- third of the number of Antarctic species belongs to the families Limopsidae, Philobryidae, and Cyamiidae. Interestingly, on the Brazilian coast, Nuculanoidea and Cuspidarioidea are more diverse in deeper waters. It has long been known that the Antarctic species of Bivalvia have very extensive depth ranges (Dell 1972, 1990), which is probably related to the glaciations occurred during the Late Caenozoic (Brey et al. 1996). Therefore, a comparison between the Brazilian and Antarctic faunas would be more complete if all the species were included, not only those from shallow waters. Resolution of the many existing taxonomic problems is also desirable, including all the levels (species, genus, families, and so on), especially for Nuculanoidea, Lucinoidea, and Galeommatoidea, which have many species with small shells, few characters, and subtle differences between them, obscuring a probable higher diversity of these groups in both regions. Almost surprisingly, three species of Bivalvia are recorded from both Brazilian and Antarctic shallow waters, which is probably related to taxonomic problems. Anyway, Brazilian malacologists are now in the process of studying deep-water $(>1000 \mathrm{~m})$ species of Bivalvia (Domaneschi \& Lopes 1990, Absalão et al. 2003, Oliveira \& Absalão 2007, 2008, 2009, 2010a, b, Passos \& Birman 2009, Simone \& Cunha $2008 \mathrm{a}, \mathrm{b})$, with the prospect of a more-detailed picture of the entire fauna in the near future.

A simple comparison between two faunas, based only on the number of species, may obscure the ecological importance of some species, especially for the Antarctic shallow-water ecosystem. Clear examples are Laternula elliptica and Adamussium colbecki, which are very abundant in some locations (e.g., Ralph \& Maxwell 1977, Arnaud et al. 1986, Berkman 1990, Nigro 1993, Cattaneo-Vietti et al. 1997, Urban \& Mercuri 1998, Mercuri et al. 1998, Passos et al. 2005, Kang et al. 2009), although they belong to groups with few representatives (Thracioidea and Pectinoidea, with two and three species, respectively). There are also species that occur in highdensity patches, such as Mysella charcoti, Lissarca miliaris, and L. notocardensis (Richardson 1980, Arnaud et al. 1986, Brey \& Hain 1992, Prezant et al. 1992, Brey et al. 1993), and other ones that are rich in biomass, e.g., Yoldia eightsi (Peck \& Bullough 1993, Peck et al. 2000), which is also ecologically important. Other important characteristics of these faunas may be also neglected when comparisons are restricted to the number of species, such as those related to the shells or to the biology of the constituent species. For example, Antarctic bivalves have in general small-sized (shorter than $15.0 \mathrm{~mm}$ ), thin, and chalky shells, without bright colors and ornamentation, as noted by Nicol (1964b, 1965, 1966a, 1967, 1970). Probably related to the seasonality of the polar environment, many species of Antarctic Bivalvia have non-pelagic development, incubating their young within the mantle cavity (Hain \& Arnaud 1992, Passos \& Domaneschi 2009). Knowledge on the biology of Antarctic Bivalvia is still restricted to a few species.
A comparison such as this, between two very different faunas of Bivalvia, has never been made previously. Antarctic and Brazilian environments have abiotic characteristics that are obviously very different, and are strongly linked to divergences in the composition, distribution, and abundance of their living organisms. Only through the analysis of their species' component species, one can point out groups that are characterized as taxonomically diverse in one fauna or another, and then emphasize studies on their ecology, eventually using them as model or monitoring organisms. This exercise raises important questions for future studies on taxonomy and biogeography as, for example, those related to changes in the composition and bathymetric distribution through a latitudinal gradient, as has been argued for both the northern and southern hemispheres, strongly evidenced by the bivalve faunas of Bivalvia (Stehli et al. 1967, Crame 2000). Crame (2000) stressed some of the determining factors in these gradients, such as the geographical distribution of coral reefs, which provide microhabitats for a more diversified fauna. He also stressed that the Brazilian fauna is incompletely understood, and therefore an existing gradient in the western Atlantic must be carefully analyzed. The present contribution aims to start this discussion for the eastern South American coast, stimulating studies on changes occurring in the composition and characteristics of the bivalve faunas from Brazil, Uruguay, Argentina, and Antarctica.

\section{Acknowledgements}

Our special thanks are due to Prof. Dr. L.R.L. Simone, MZUSP, who kindly provided bibliographic material and shared opinions about species from Brazil; to Diego Zelaya, who discussed some of the ideas contained in the text; and to CNPq and PPG of Ecologia (UNICAMP) for financial support and a scholarship.

\section{References}

ABBOTT, R.T. 1974. American Seashells. 2 ed. Van Nostrand Reinhold Co., New York.

ABELE, D., TESCH, C., WENCKE, P. \& PÖRTNER, H.O. 2001. How does oxidative stress relate to thermal tolerance in the Antarctic bivalve Yoldia eightsi? Antarct. Sci. 13:11-118.

ABSALÃO, R.S. \& PIMENTA, A.D. 2005. Moluscos marinhos da APA do Arquipélago de Santana, Macaé, RJ. Editora Ciência Moderna, Rio de Janeiro.

ABSALÃO, R.S., CAETANO, C.H.S. \& PIMENTA, A.D. 2003. Novas ocorrências de gastrópodes e bivalves marinhos do Brasil (Mollusca). Rev. Bras. Zool. 20:323-328.

AHN, I.Y. 1997. Some ecological and physiological strategies for energy conservation of the Antarctic clam, Laternula elliptica. Kor. J. Polar Res. 8:77-83.

ALDEA, C. \& TRONCOSO, J.S. 2008. Systematics and distribution of shelled molluscs (Gastropoda, Bivalvia and Scaphopoda) from the South Shetland Islands to the Bellingshausen Sea, West Antarctica. Iberus 26:43-117.

ALDEA, C., OLABARRIA, C. \& TRONCOSO, J.S. 2008. Bathymetric zonation and diversity gradient of gastropods and bivalves in West Antarctica from the South Shetland Islands to the Bellingshausen Sea. Deep-Sea Res. Pt. I 55:350-368.

ALDEA, C., OLABARRIA, C. \& TRONCOSO, J.S. 2009. Spatial patterns of benthic diversity in molluscs from West Antarctica. Antarct. Sci. 21:341-353.

ALLEN, J.A. 2004. The Recent species of the genera Limatula and Limea (Bivalvia, Limacea) present in the Atlantic, with particular reference to those in deep water. J. Nat. Hist. 38:2591-2653.

AMARAL, A.C.Z., RIZZO, A.E. \& ARRUDA, E.P. 2006. Manual de identificação dos invertebrados marinhos da região sudeste-sul do Brasil. Editora USP, São Paulo, v.1. 
ARNAUD, P.M. 1972. Sur une petite collection de gastéropodes prosobranches et pélécypodes de l'Ile Petermann (Antarctique). Téthys 4:429-436.

ARNAUD, P.M. 1973. Invertébrés marins des XIIème et XVème Expéditions Antarctiques Françaises en Terre Adélie. 13. Polyplacophores, Scaphopodes et Pélécypodes. Téthys 5:549-560.

ARNAUD, P.M. \& HAIN, S. 1992. Quantitative distribution of the shelf and slope molluscan fauna (Gastropoda, Bivalvia) of the eastern Weddell Sea (Antarctica). Polar Biol. 12:103-109.

ARNAUD, P.M., JAZDZEWSKIK, K., PRESLER, P. \& SICINSKI, J. 1986. Preliminary survey of benthic invertebrates collected by Polish Antarctic expeditions in Admiralty Bay (King George Island, South Shetlands Islands, Antarctica). Pol. Polar Res. 7:7-24.

ARNAUD, P.M., TRONCOSO, J.S. \& RAMOS, A. 2001. Species diversity and assemblages of macrobenthic Mollusca from the South Shetland Islands and Bransfield Strait (Antarctica). Polar Biol. 24:105-112.

ARRUDA, E.P. 2005. Mollusca Bivalvia (Veneroida) da costa sudeste do Brasil. PhD Thesis, University of São Paulo, São Paulo.

ARRUDA, E.P. \& DOMANESCHI, O. 2005. New species of Macoma (Bivalvia: Tellinoidea: Tellinidae) from southeastern Brazil, and with description of its gross anatomy. Zootaxa 1012:13-22.

ARRUDA, E.P., DOMANESCHI, O., FRANCISCO, J.A. \& BARROS, J.C.N. 2007. Corbula tarasconii, a new species of Corbulidae (Mollusca, Bivalvia) from offshore Brazil. Nautilus 121:201-209.

AVELAR, W.E.P. \& NARCHI, W. 1984. Anatomia funcional de Brachidontes darwinianus darwinianus (Orbigny, 1846) (Mollusca: Bivalvia). Pap. Avulsos Zool. 35:331-359.

BEESLEY, P.L., ROSS, G.J.B. \& WELLS, A. 1998. Mollusca: the Southern Synthesis. Fauna of Australia. CSIRO Publishing, Melbourne, v.5, part A.

BERKMAN, P.A. 1990. The population biology of the Antarctic scallop Adamusium colbecki (Smith, 1902) at New Harbour, Ross Sea. In Antarctic ecosystems: ecological change and conservation. Proceedings of the Fifth SCAR Biological Symposium (K.R. Kerry \& G. Hempel, eds.). Springer-Verlag, Berlin, p.281-288.

BERKMAN, P.A., WALLER, T.R. \& ALEXANDER, S.P. 1991. Unprotected larval development in the Antarctic scallop Adamussium colbecki (Mollusca: Bivalvia: Pectinidae). Antarct. Sci. 3:151-157.

BIGATTI, G., PENCHASZADEH, P.E. \& MERCURI, G. 2001. Aspects of the gonadal cycle in the Antarctic bivalve Laternula elliptica. J. Shellfish Res. 20:283-287.

BOSS, K.J. 1966. The subfamily Tellininae in the western Atlantic. The genus Tellina. Johnsonia 4:217-272.

BOSS, K.J. 1968. The subfamily Tellininae in the western Atlantic. The genus Tellina (part II) and Tellidora. Johnsonia 4:273-344.

BOSS, K.J. 1969. The subfamily Tellininae in the western Atlantic. The genus Strigilla. Johnsonia 4:345-366.

BOSS, K.J. 1972. The genus Semele in the western Atlantic. Johnsonia 5:1-32.

BOSS, K.J. 1982. Mollusca. In Synopsis and classification of living organisms (S.P. Parker, ed.). McGraw-Hill, New York, v.1.

BRANDT, A., LINSE, K. \& MÜHLENHARDT-SIEGEL, U. 1999. Biogeography of Crustacea and Mollusca of the Subantarctic and Antarctic regions. Sci. Mar. 63(suppl.1):383-389.

BREY, T. \& HAIN, S. 1992. Growth, reproduction and production of Lissarca notorcadensis (Bivalvia: Philobryidae) in the Weddell Sea, Antarctica. Mar. Ecol.-Prog. Ser. 82:219-226.

BREY, T., STARMANS, A., MAGIERA, U. \& HAIN, S. 1993. Lissarca notorcadensis (Bivalvia: Philobryidae) living on Notocidaris sp. (Echinoidea: Cidaridae): population dynamics in limited space. Polar Biol. 13:89-95.

BREY, T., DAHM, C., GORNY, M., KLAGES, M., STILLER, M. \& ARNTZ, W.E. 1996. Do Antarctic invertebrates show an extended level of eurybathy? Antarct. Sci. 8:3-6.

CARCELleS, A.R. 1944. Catálogo de los moluscos marinos de Puerto Quequén. Revta. Mus. La Plata, N.S., Zool. 3:233-309.
CARCELLES, A.R. 1950. Catálogo de los moluscos marinos de Patagonia. An. Museo Nahuel Huapí 2:41-99.

CARCELLES, A.R. 1953. Catálogo de la Malacofauna Antártica Argentina. An. Museo Nahuel Huapí 3:150-250.

CARCELLES, A.R. \& WILLIAMSON, S.I. 1951. Catálogo de los Moluscos marinos de la Provincia Magallanica. Rev. Inst. Invest. Mus. Argent. Cienc. Nat. Zool. 2:225-383.

CASTELLANOS, Z.J.A. 1963. Adiciones a la Malacofauna Antartica. Contr. Inst. Antart. Argent. 80:1-11.

CATtAneO-Vietti, R., ChiAntore, M. \& Albertelli, G. 1997. The population structure and ecology of the Antarctic scallop Adamussium colbecki (Smith, 1902) at Terra Nova Bay (Ross Sea, Antarctica). Sci. Mar. 61(suppl.2):15-24.

CATTANEO-VIETTI, R., CHIANTORE, M., SCHIAPARELLI, S. \& ALBERTELLI, G. 2000. Shallow- and deep-water mollusc distribution at Terra Nova Bay (Ross Sea, Antarctica). Polar Biol. 23:173-182.

CHIANTORE, M., CATTANEO-VIETTI, R. \& HEILMAYER, O. 2003. Antarctic scallop (Adamussium colbecki) annual growth rate at Terra Nova Bay. Polar Biol. 26:416-419.

CLARKE, A. 1983. Life in cold water: the phisiological ecology of polar ectotherms. Oceanogr. Mar. Biol. 21:241-453.

COAN, E.V., SCOTT, P.V. \& BERNARD, F.R. 2000. Bivalve Seashells of Western North America. Marine Bivalve Mollusks from Arctic Alaska to Baja California. Santa Barbara Museum of Natural History, Santa Barbara.

COELHO, A.C.S. \& CAMPOS, D.R.B. 1975. Contribuições ao conhecimento dos moluscos do Rio de Janeiro, Brasil. Arq. Mus. Nac. RJ 55:35-57.

CRAME, J.A. 1992. Evolutionary history of the Polar Regions. Hist. Biol. 6:37-60.

CRAME, J.A. 1993. Bipolar mollusks and their evolutionary implications. J. Biogeogr. 20:145-161.

CRAME, J.A. 1996. Evolution of high-latitude molluscan faunas. In Origin and evolutionary radiation of the Mollusca (J.D. Taylor, ed.). Oxford University Press, Oxford, p.119-131.

CRAME, J.A. 1997. An evolutionary framework for the polar regions. J. Biogeogr. 24:1-9.

CRAME, J.A. 2000. Evolution of taxonomic diversity gradients in the marine realm: evidence from the composition of Recent bivalve faunas. Paleobiology 26:188-214.

DELL, R.K. 1964. Antarctic and Subantarctic Mollusca: Amphineura, Scaphopoda and Bivalvia. Discovery Rep. 33:93-250.

DELL, R.K. 1972. Antarctic benthos. Adv. Mar. Biol. 10:1-216.

DELL, R.K. 1990. Antarctic Mollusca. With special reference to the fauna of the Ross Sea. R. Soc. N.Z. Bull. 27:1-311.

DENADAI, M.R., ARRUDA, E.P., DOMANESCHI, O. \& AMARAL, A.C.Z. 2006. Veneridae (Mollusca, Bivalvia) da costa norte do Estado de São Paulo, Brasil. Biota Neotrop.: http://www.biotaneotropica.org.br/v6n3/ pt/abstract?inventory+bn01106032006.

DOMANESCHI, O. 1984. Família Myidae Lamarck, 1809. Inf. SBMa 40:9-12.

DOMANESCHI, O. 1985. Família Psammobiidae Fleming, 1828. (Partes I, II, III). Inf. SBMa 47:9-12; 48:9-12; 49:9-12.

DOMANESCHI, O. \& LOPES, S.G.B.C. 1986a. Família Pinnidae Leach, 1819. Inf. SBMa 53:9-12; 54:9-12.

DOMANESCHI, O. \& LOPES, S.G.B.C. 1986b. Família Pteriidae Gray 1847. Inf. SBMa 57:9-12; 58:9-12.

DOMANESCHI, O. \& LOPES, S.G.B.C. 1988/9. Família Donacidae Fleming, 1828. Inf. SBMa 87:9-12; 88:9-12; 92:9-12; 93:9-12.

DOMANESCHI, O. \& LOPES, S.G.B.C. 1990. Calyptogena (Calyptogena) birmani, a new species of Vesicomyidae from Brazil. Malacologia 31:363-370 
DOMANESCHI, O. \& MARTINS, C.M. 2002. Isognomon bicolor (C. B. Adams) (Bivalvia, Isognomonidae): primeiro registro para o Brasil, redescrição da espécie e considerações sobre a ocorrência e distribuição de Isognomon na costa brasileira. Rev. Bras. Zool. 19:611-627.

DOMANESCHI, O. \& NARCHI, W. 1998. Adaptive convergences in two nestling bivalves (Myoidea: Myidae: Hiatellidae) of the Brazilian littoral. Iheringia Sér. Zool. 85:89-96.

DOMANESCHI, O., NARCHI, W., LOPES, S.G.B.C., MESERANI, G.L.C., PASSOS, F.D., SARTORI, A.F., PANTALEÃO, C., BRANCATTO, C. \& BIBANCOS, M. 2007. Bivalves antárticos e subantárticos: projetos, experiências na Antártica e resultados obtidos pelos malacólogos do Instituto de Biociências da Universidade de São Paulo dentro do PROANTAR - Programa Antártico Brasileiro. In Tópicos em Malacologia: Ecos do XVIII Encontro Brasileiro de Malacologia (S.B. Santos, A.D. Pimenta, S.C. Thiengo, M.A. Fernandez \& R.S. Absalão, orgs.). SBMa, Rio de Janeiro, p.41-59.

DOMANESCHI, O. \& SHEA, E.K. 2004. Shell morphometry of Western Atlantic and Indo-West Pacific Asaphis; functional morphology and ecological aspects of Asaphis deflorata from Florida Keys, U.S.A. (Bivalvia: Psammobiidae). Malacologia 46:249-275.

DOMANESCHI, O., SILVA, J.R.M.C., PORTO NETO, L.R. \& PASSOS, F.D. 2002. New perspectives on the dispersal mechanisms of the Antarctic brooding bivalve Mysella charcoti (Lamy, 1906). Polar Biol. 25:538-541.

ESTEVES, I.R.F. 1984. Recent bivalves (Paleotaxodonta and Pteriomorpha) from the Brazilian continental shelf. Pesq. Porto Alegre 16:190-226.

FORCELLI, D.O. 2000. Moluscos Magallánicos. Guía de los moluscos de la Patagônia y del Sul de Chile. Vázquez Mazzini Editores, Buenos Aires.

GRIFFITHS, H.J., LINSE, K. \& CRAME, J.A. 2003. SOMBASE - Southern Ocean Mollusc Database: a tool for biogeographic analysis in diversity and ecology. Org. Divers. Evol. 3:207-213.

GUERÓN, C.O.C. \& COELHO, A.C.S. 1989. Considerações taxonômicas e morfologia de Dosinia (Dosinia) concentrica (Born, 1778) (Mollusca, Bivalvia, Veneridae). Bol. Mus. Nac., Zool. 334:1-19.

HAIN, S. 1990. Die beschalten benthischen Mollusken (Gastropoda und Bivalvia) des Weddellmeeres, Antarktis. Ber. Polarforsch. 70:1-184.

HAIN, S. \& ARNAUD, P.M. 1992. Notes on the reproduction of high-Antarctic molluscs from the Weddell Sea. Polar Biol. 12:303-312.

HEDLEY, C. 1916. Mollusca. Austral. Ant. Exp. 1911-1914, Sci. Rep. Series C 4:3-80.

HEILMAYER, O. \& BREY, T. 2003. Saving by freezing? Metabolic rates of Adamussium colbecki in a latitudinal context. Mar. Biol. (Berlin) 143:477-484.

KANG, D.H., AHN, I.Y. \& CHOI, K.S. 2009. The annual reproductive pattern of the Antarctic clam, Laternula elliptica from Marian Cove, King George Island. Polar Biol. 32:517-528.

KLAPPENBACH, M.A. 1965. Lista preliminar de los Mytilidae brasileños com claves para su determinación y notas sobre su distribuición. An. Acad. Bras. Ciênc. 37(supl.):327-352.

KLAPPENBACH, M.A. 1966a. Um nuevo representante del género Cosa obtenido em aguas brasilenas. Rev. Bras. Biol. 26:23-27.

KLAPPENBACH, M.A. 1966b. Nueva especie de Modiolus (Mollusca, Pelecypoda) de la costa brasileña. Pap. Avulsos Zool. 19:251-257.

KNUDSEN, J. 1970. The systematics and biology of abyssal and hadal Bivalvia. Galathea Rep. 11:7-241.

LAMY, E. 1906a. Lamellibranches recueillis par l'Expédition Antarctique Française du Dr. Charcot. Bull. Mus. Natl. Hist. Nat. 12:44-52.

LAMY, E. 1906b. Expédition Antarctique Française (1903-1904) commandée par le Dr. Jean Charcot. Gastropodes Prosobranches et Pélécypodes. Sci. Nat. Doc. Scientif. 1-20.

LAMY, E. 1911. Deuxième Expédition Antarctique Française (1908-1910) commandée par le Dr. Jean Charcot. Gastropodes Prosobranches, Scaphopode et Pélécypodes. Sci. Nat. Doc. Scientif. 1-32.

LEONEL, R.M.V., LOPES, S.B.G.C. \& AVERSARI, M. 2002. Distribution of wood-boring bivalves in the Mamanguape River estuary, Paraíba, Brazil. J. Mar. Biol. Assoc. UK 82:1039-1040.
LINSE, K. 1999. Mollusca of the Magellan region. A checklist of the species and their distribution. Sci. Mar. 63(supl.1):399-407.

LINSE, K. 2004. Scotia Arc deep-water bivalves: composition, distribution and relationship to the Antarctic shelf fauna. Deep-Sea Res. Pt. II 51:1827-1837.

LINSE, K. 2006. New records of shelled marine molluscs at Bouvet Island and preliminary assessment of their biogeographic affinities. Polar Biol. 29:120-127.

LOPES, S.G.B.C. \& NARCHI, W. 1993. Levantamento e distribuição das espécies de Teredinidae (Mollusca - Bivalvia) no manguezal da Praia Dura, Ubatuba, São Paulo, Brasil. Bol. Inst. Oceanogr. 41:29-38.

MARINI, A.C. 1974. O gênero Verticordia Wood, 1844 (Bivalvia, Verticordiidae) na plataforma continental brasileira. Pap. Avulsos Zool. 28:241-244.

MATTHEWS, H.R. \& KEMPF, M. 1970. Moluscos marinhos do Norte e Nordeste do Brasil. II-Moluscos do Arquipélago de Fernando de Noronha. Arq. Cienc. Mar. 10:1-53.

MELVILL, J.C. \& STANDEN, R. 1907. The marine Mollusca of the Scotish National Antarctic Expedition. Trans. R. Soc. Edinb. 46:119-157.

MERCURI, G., IKEN, K., LEDESMA, B. \& DUBOIS, R.F. 1998. On the distribution patterns and density of the Antarctic infaunal bivalve Laternula elliptica in Potter Cove, King George Island, Antarctica. Ber. Polarforsch. 299:137-143.

MESERANI, G.L.C., DOMANESCHI, O. \& LOPES, S.G.B.C. 2004. Functional anatomy of feeding in Psiloteredo healdi (Teredinidae): how the animal handles two discrete food sources. American Malacological Bulletin 18:143-154.

MIKKELSEN, P.M. \& BIELER, R. 2008. Seashells of southern Florida: living marine mollusks of the Florida Keys and adjacent regions. Princeton University Press, New Jersey.

MORRISON, J.P.E. 1971. Western Atlantic Donax. Proc. Biol. Soc. Wash. 83:545-568.

MÜHLENHARDT-SIEGEL, U. 1989. Antarktische Bivalvia der Reisen des FS "Polarstern" und des FFS "Walther Herwig" aus den Jahren 1984 bis 1986. Mitt. Hamb. Zool. Mus. Inst. 86:153-178.

NARCHI, W. 1966. The functional morphology of Ceratobornia cema, new species of Erycinacea (Mollusca, Eulamellibranchiata). An. Acad. Bras. Ciênc. 38:513-524.

NARCHI, W. 1973. On the functional morphology of Hiatella solida (Hiatellidae: Bivalvia). Mar. Biol. (Berlin) 19:332-337.

NARCHI, W. 1974. Functional morphology of Petricola (Rupellaria) typica (Bivalvia: Petricolidae). Mar. Biol. (Berlin) 27:123-129.

NARCHI, W. 1975. Functional morphology of new Petricola (Mollusca Bivalvia) from the littoral of São Paulo, Brazil. Proc. Malacol. Soc. Lond. 41:451-465.

NARCHI, W. 1976. Meiocardia agassizii Dall, 1886 in the Brazilian Littoral (Mollusca - Bivalvia). Stud. Neotrop. Fauna Environ. 11:205-210.

NARCHI, W. 1985a. Família Glossidae Gray, 1847. Inf. SBMa 45:9-12.

NARCHI, W. 1985b. Família Leptonidae Gray, 1847. Inf. SBMa 52:9-12.

NARCHI, W. \& DOMANESCHI, O. 1977. Semele casali Doello-Jurado, 1949 (Mollusca-Bivalvia) in the Brazilian Littoral. Stud. Neotrop. Fauna Environ. 12:263-272.

NARCHI, W., DOMANESCHI, O. \& PASSOS, F.D. 2002. Bivalves Antárticos e Subantárticos coletados durante as Expedições Científicas Brasileiras à Antártica I a IX (1982-1991). Rev. Bras. Zool. 19:645-675.

NICOL, D. 1964a. Lack of shell-attached pelecypods in Arctic and Antarctic waters. Nautilus 77:92-93.

NICOL, D. 1964b. An essay on size of marine pelecypods. J. Paleontol. 38:968-974.

NICOL, D. 1965. Ecologic implications of living pelecypods with calcareous spines. Nautilus 78:109-116.

NICOL, D. 1966a. Size of pelecypods in recent marine faunas. Nautilus 79:109-113. 
NICOL, D. 1966b. Descriptions, ecology and geographic distribution of some Antarctic pelecypods. Bull. Am. Paleontol. 51:1-102.

NICOL, D. 1967. Some characteristics of cold-water marine pelecypods. J. Paleontol. 41:1330-1340.

NICOL, D. 1970. Antarctic pelecypod faunal peculiarities. Science 168:12481249.

NICOL, D. \& GAVENDA, A. P. 1964. Inferences derived from the general analysis of Recent and fossil marine pelecypod faunas. J. Paleontol. 38:975-983.

NIGRO, M. 1993. Nearshore population characteristics of the circumpolar Antarctic scallop Adamussium colbecki (Smith, 1902) at Terra Nova Bay (Ross Sea). Antarct. Sci. 5:377-378.

OLIVEIRA, C.D.C. \& ABSALÃO, R.S. 2007. Primeiro registro de Mendicula ferruginosa, Kelliella atlantica e Lyonsiella subquadrata (Mollusca, Pelecypoda) para águas brasileiras. Biociências 15:63-67.

OLIVEIRA, C.D.C. \& ABSALÃO, R.S. 2008. The genera Limatula and Limea (Mollusca, Pelecypoda, Limidae) from deep waters off Brazil. Zootaxa 1940:48-58.

OLIVEIRA, C.D.C. \& ABSALÃO, R.S. 2009. The genera Myonera, Octoporia, and Protocuspidaria (Pelecypoda, Cuspidariidae) from deep waters of Campos Basin, Rio de Janeiro, Brazil with descriptions of two new species. Am. Malacol. Bull. 27:141-156.

OLIVEIRA, C.D.C. \& ABSALÃO, R.S. 2010a. Review of the Septibranchia (Pelecypoda: Mollusca) from deep sea of Campos Basin, Brazil: family Verticordiidae, with description of a new species. J. Mar. Biol. Assoc. UK 90:809-817.

OLIVEIRA, C.D.C. \& ABSALÃO, R.S. 2010b. Review of the Septibranchia (Mollusca: Pelecypoda) from the deep sea of Campos Basin, Brazil: family Lyonsiellidae, with description of a new species. Sci. Mar. 74:305-316.

PASSOS, F.D. \& BIRMAN, A. 2009. New records of Bentharca asperula (Dall, 1881) (Mollusk, Bivalvia, Arcidae) from Brazil. Biota Neotrop. 9(4): http://www.biotaneotropica.org.br/v9n4/en/abstract?shortcommun ication+bn02309042009.

PASSOS, F.D. \& DOMANESCHI, O. 2006. A new species of Mysella Angas, 1877 (Bivalvia: Galeommatoidea) from Admiralty Bay, King George Island, South Shetlands, Antarctica, with data on its biology and functional anatomy. Polar Biol. 29:389-398.

PASSOS, F.D. \& DOMANESCHI, O. 2009. The anatomical characters related to the brooding behavior of two Antarctic species of Mysella Angas, 1877 (Bivalvia, Galeommatoidea, Lasaeidae), with direct and indirect evidences of ovoviviparity. Polar Biol. 32:271-280.

PASSOS, F.D., DOMANESCHI, O. \& SARTORI, A.F. 2005. Biology and functional morphology of the pallial organs of the Antarctic bivalve Mysella charcoti (Lamy, 1906) (Galeommatoidea: Lasaeidae). Polar Biol. 28:372-380.

PASSOS, F.D., MESERANI, G.L.C. \& GROS, O. 2007. Structural and ultrastructural analysis of the gills of the bacterial-bearing bivalve Thyasira falklandica Smith, 1885). Zoomorphology 126:153-162.

PECK, L.S. \& BULLOUGH, L.W. 1993. Growth and population structure in the infaunal bivalve Yoldia eightsi in relation to iceberg activity at Signy Island, Antarctica. Mar. Biol. (Berlin) 117:235-241.

PECK, L.S., COLMAN, J.G. \& MURRAY, A.W.A. 2000. Growth and tissue mass cycles in the infaunal bivalve Yoldia eightsi at Signy Island, Antarctica. Polar Biol. 23:420-428.

PECK, L.S. \& CONWAY, L.Z. 2000. The myth of metabolic cold adaptation: oxygen consumption in stenothermal Antarctic bivalves. In The Evolutionary Biology of the Bivalvia (E.M. Harper, J.D. Taylor \&, J.A. Crame, eds.). Geological Society, London, Special Publications 177:441-450.

PECK, L.S., POWELL, D.K. \& TYLER, P.A. 2007. Very slow development in two Antarctic bivalve molluscs, the infaunal clam Laternula elliptica and the scallop Adamussium colbecki. Mar. Biol. (Berlin) 150:1191-1197.

PELSENEER, P. 1903. Mollusques (Amphineures, Gastropodes et Lamellibranches). Expéd. Antarct. Belgue 1-85.
PENNA, L. 1971. Novas espécies e registros de Limidae e Carditidae (Pelecypoda) no litoral brasileiro. Pap. Avulsos Zool. 24:155-159.

PENNA-NEME, L. 1978. Os Glycymerididae da costa brasileira (Mollusca, Bivalvia). Pap. Avulsos Zool. 32:59-70.

PENNA-NEME, L. 1983. Família Glycymerididae Newton, 1922. Inf. SBMa 28:9-12.

PENNA-NEME, L. \& CRUZ-NATALI, M.L. 1984. Phacoides (Parvilucina) coupoisi Klein, 1967, novo sinônimo de Codakia (Ctena) pectinella (C. B. Adams, 1852), e algumas considerações sobre os Lucinidae brasileiros (Mollusca, Bivalvia). Pap. Avulsos Zool. 35:153-158.

POWELL, A.W.B. 1951. Antarctic and Subantarctic Mollusca: Pelecypoda and Gastropoda. Discovery Rep. 26:47-196.

POWELL, A.W.B. 1958. Mollusca from the Victoria-Ross Quadrants of Antarctica. B.A.N.Z.A.R.E. Rep. (Ser.B) 6:165-216.

POWELL, A.W.B. 1960. Antarctic and Subantarctic Mollusca. Rec. Auck. Inst. Mus. 5:117-193.

PREZANT, R.S., SHOWERS, M., WINSTEAD, R.L. \& CLEVELAND, C. 1992. Reproductive ecology of the Antarctic bivalve Lissarca notorcadensis (Philobryidae). Am. Malacol. Bull. 9:173-186.

QUAST, M.P. 2003. Moluscos Bivalves (Arcoida e Ostreoida) da Costa Sudeste do Brasil. Master's Thesis, University of Campinas, Campinas.

RABARTS, I.W. \& WHYBROW, S. 1979. A revision of the Antarctic and Subantarctic members of the genus Yoldia Möller, 1842 (Bivalvia: Nuculanidae). J. Nat. Hist. 13:161-183.

RALPH, R. \& MAXWELL, J.G.H. 1977. Growth of two Antarctic lamellibranchs: Adamussium colbecki and Laternula elliptica. Mar. Biol. (Berlin) 42:171-175.

RESGALLA, C., WEBER, L.I. \& CONCEIÇÃO, M.B. 2008. O mexilhão Perna perna (L.): biologia, ecologia e aplicações. Interciência, Rio de Janeiro.

RICHARDSON, M.G. 1980. The ecology and reproduction of the brooding Antarctic bivalve Lissarca miliaris. Brit. Antarct. Surv. B. 49:91-115.

RIOS, E.C. 1994. Seashells of Brazil. 2 ed. Fundação Universidade do Rio Grande, Rio Grande.

RIOS, E.C. 2009. Compendium of Brazilian Sea Shells. Evangraf, Rio Grande.

SALVADOR, L.B., DOMANESCHI, O., AMARAL, A.C.Z., MORGADO, E.H. \& HENRIQUES, S.A. 1998. Malacofauna da região entremarés de praias da Ilha de São Sebastião (São Paulo, Brasil). Rev. Bras. Zool. 15:1013-1035.

SARTORI, A.F. \& DOMANESCHI, O. 2005. The functional morphology of the Antarctic bivalve Thracia meridionalis Smith, 1885 (Anomalodesmata: Thraciidae). J. Molluscan Stud. 71:199-210.

SARTORI, A.F., PASSOS, F.D. \& DOMANESCHI, O. 2006. Arenophilic mantle glands in the Laternulidae (Bivalvia: Anomalodesmata) and their evolutionary significance. Acta Zool., Stockholm 87:265-272.

SIMONE, L.R.L. 1998. A new species of Gari (Gobeus) (Bivalvia, Tellinoidea, Psammobiidae) from Bahia coast, Brazil. J. Conchol. 36:35-38.

SIMONE, L.R.L. 2001. Revision of the genus Parabornia (Bivalvia: Galeommatoidea: Galeommatidae) from the Westerns Atlantic, with description of a new species from Brazil. J. Conchol. 37:159-169.

SIMONE, L.R.L. 2008. A new species of Chlamydoconcha Dall, 1884, from southeastern Brazil (Bivalvia: Chlamydoconchidae). Nautilus 122:252-258.

SIMONE, L.R.L. 2009a. Comparative morphology among representatives of main taxa of Scaphopoda and basal protobranch Bivalvia (Mollusca). Pap. Avulsos Zool. 49:405-457.

SIMONE, L.R.L. 2009b. A new species of Acar (Bivalvia, Arcidae) from São Pedro e São Paulo Archipelago, Brazil. Novapex 10:9-16.

SIMONE, L.R.L. \& CHICHVARKHIN, A. 2004. Comparative morphological study of four species of Barbatia occurring on the southern Florida coast (Arcoidea, Arcidae). Malacologia 46:355-379.

SIMONE, L.R.L. \& CUNHA, C.M. 2008a. Revision of the genus Spinosipella (Bivalvia: Verticordiidae), with descriptions of two new species from Brazil. Nautilus 122:57-78. 
SIMONE, L.R.L. \& CUNHA, C.M. 2008b. Supplementary data from a recent revision of the genus Spinosipella (Bivalvia, Septibranchia). Strombus 15:8-14.

SIMONE, L.R.L. \& GONÇALVES, E.P. 2006. Anatomical study on Myoforceps aristatus, an invasive boring bivalve in S. E. Brazilian Coast (Mytilidae). Pap. Avulsos Zool. 46:57-65.

SIMONE, L.R.L. \& PENCHASZADEH, P.E. 2008. Redescription of Hiatella meridionalis d’Orbigny, 1846 (Mollusk, Hiatellidae) from Argentina. Pap. Avulsos Zool. 48:119-127.

SMITH, E.A. 1915. Mollusca. Part I. Gastropoda, Prosobranchia, Scaphopoda, and Pelecypoda. Nat. Hist. Rep. Br. Antarct. "Terra Nova” Exped. Zool. 2:61-112.

SOARES-GOMES, A. \& PIRES-VANIN, A.M.S. 2003. Padrões de abundância, riqueza e diversidade de moluscos bivalves na plataforma continental ao largo de Ubatuba, São Paulo, Brasil: uma comparação metodológica. Rev. Bras. Zool. 20:717-725.

SOOT-RYEN, T. 1951. Antarctic pelecypodes. Scient. Res. Norw. Antarct. Exped. 1927-1928 32:1-46.

SOUZA, J.R.B., ROCHA, C.M.C. \& LIMA, M.P.R. 2005. Ocorrência do bivalve exótico Mytilopsis leucophaeta (Conrad) (Mollusca, Bivalvia), no Brasil. Rev. Bras. Zool. 22:1204-1206.

STEHLI, F.G., McALESTER, A.L. \& HESLEY, C.E. 1967. Taxonomic diversity of recent bivalves and some implications for geology. Geol. Soc. Am. Bull. 78:455-466.

STOCKTON, W.L. 1984. The biology and ecology of the epifaunal scallop Adamussium colbecki on the west side of McMurdo Sound, Antarctica. Mar. Biol. (Berlin) 78:171-178.

STUARDO, J. 1962. Sobre el género Limopsis y la distribuición de $L$. jousseaumei (Mabille y Rochebrune, 1889) (Mollusca: Bivalvia). Gayana 6:5-10.
TENÓRIO, D.O. 1984. O gênero Tellina Linnaeus, 1758 (Mollusca, Bivalvia) na plataforma continental brasileira. Trab. Oceanogr. 18:7-138.

TENÓRIO, D.O., MELLO, R.L. \& SILVA, O.C. 1986. O gênero Macoma Leach, 1819 (Bivalvia Tellinidae) na plataforma continental brasileira. Cad. Ômega, Ser. Cienc. Aquat. 2:7-39.

THIELE, J. 1912. Die Antarktischen Schnecken und Muscheln. Dt. Südpol.Exped. 1901-1903 XIII Zool. 5:183-285.

TRONCOSO, J. \& ALDEA, C. 2008. Macrobenthic mollusc assemblages and diversity in the West Antarctica from the South Shetland Islands to the Bellingshausen Sea. Polar Biol. 31:1253-1265.

URBAN, H.J. \& MERCURI, G. 1998. Population dynamics of the bivalve Laternula elliptica from Potter Cove, King George Island, South Shetland Islands. Antarct. Sci. 10:153-160.

VILLARROEL, M. \& STUARDO, J. 1998. Protobranchia (Mollusca: Bivalvia) chilenos recientes y algunos fossiles. Malacologia 40:113-229.

ZELAYA, D.G. 2005. The bivalves from the Scotia Arc islands: species richness and faunal affinities. Sci. Mar. 69(suppl.2):113-122.

ZELAYA, D.G. 2009. The genera Thyasira and Parathyasira in the Magellan Region and adjacent Antarctic waters (Bivalvia: Thyasiridae). Malacologia 51:271-290.

ZELAYA, D.G. 2010. New species of Thyasira, Mendicula, and Axinulus (Bivalvia: Thyasiroidea) from Sub-Antarctic and Antarctic waters. Polar Biol. 33:607-616.

ZELAYA, D.G. \& ITUARTE, C. 2002. The identity of Waldo parasiticus (Dall, 1876) and description of Waldo trapezialis new species (Bivalvia: Galeommatoidea). Nautilus 116:109-117.

ZELAYA, D.G. \& ITUARTE, C. 2006. Redescription of two Antarctic species of Cuspidaria: C. concentrica Thiele, 1912 and C. minima (Egorova, 1993) (Bivalvia: Cuspidariidae). Veliger 48:170-177. 